This is the final peer-reviewed accepted manuscript of:

Zattoni, Elena, Anna Maria Perdon, and Giuseppe Conte. 2017. "Output Regulation by Error Dynamic Feedback in Hybrid Systems with Periodic State Jumps." Automatica 81 (July): 322-34. doi:.

The final published version is available online at:

https://doi.org/10.1016/i.automatica.2017.03.037

Rights / License:

The terms and conditions for the reuse of this version of the manuscript are specified in the publishing policy. For all terms of use and more information see the publisher's website.

This item was downloaded from IRIS Università di Bologna (https://cris.unibo.it/)

When citing, please refer to the published version. 


\title{
Output Regulation by Error Dynamic Feedback in Hybrid Systems with Periodic State Jumps
}

\author{
Elena Zattoni ${ }^{\mathrm{a}, \star}$, Anna Maria Perdon ${ }^{\mathrm{b}}$, Giuseppe Conte ${ }^{\mathrm{b}}$

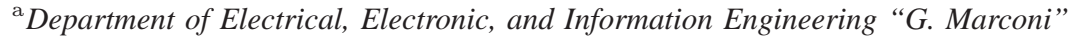 \\ Alma Mater Studiorum · University of Bologna, 40136 Bologna, Italy \\ ${ }^{\mathrm{b}}$ Department of Information Engineering, Polytechnic University of Marche, \\ 60131 Ancona, Italy
}

\begin{abstract}
This work deals with output regulation in multivariable hybrid systems featuring a continuous-time linear dynamics periodically affected by instantaneous changes of the state. More precisely, given a hybrid linear plant and a hybrid linear exogenous system, with periodic state jumps, the problem consists in finding a hybrid feedback regulator, with the same characteristics, achieving global asymptotic stability of the closed-loop dynamics and asymptotic tracking of the reference generated by the exogenous system for all the initial states. Starting from a general, necessary and sufficient condition for the existence of a solution, the discussion leads to a more specific, sufficient condition which outlines the computational framework for a straightforward synthesis of the compensator. The internal model principle is shown to hold in a more general formulation than the original one, adapted to the hybrid systems considered. A numerical example is worked out with the aim of illustrating how to implement the devised technique. The geometric approach is the key methodology in attaining these results.
\end{abstract}

Key words: hybrid systems; output regulation; global asymptotic stability; geometric approach.

\section{Introduction}

Hybrid systems with state jumps are dynamical systems which exhibit a continuous-time behavior (the so-called flow dynamics) interrupted by state discontinuities (jump dynamics). These dynamical systems have drawn an increasing amount of research effort during the last decade, mainly because they are particularly effective in representing the peculiar way some real systems, occurring in various fields of science and engineering, operate (see, e.g., Goebel et al., 2009, 2012). Indeed, many classes of jump hybrid systems can be distinguished on the basis of several characteristics, such as the flow and jump dynamics being linear or nonlinear, the jumps being time driven or state driven, and so on. Thus, the control synthesis raises a number of typical issues and requires ad-hoc devised methodologies, depending on the features of the hybrid systems addressed. In particular, this work is focused on hybrid systems with a continuoustime linear dynamics subject to periodic state jumps and investigates the output regulation problem.

Output regulation is a classic problem of control theory and it essentially consists in finding a feedback regulator which, for a given plant and a given exogenous system, ensures stability of the closed-loop dynamics and asymptotic tracking of the reference generated by the exogenous system for all the initial states. A less basic formulation of this problem (including decoupling of a disturbance generated by the exogenous system and directly affecting the plant) has been studied for scalar hybrid systems with periodic state jumps in (Marconi and Teel, 2010, 2013) and for multivariable hy-

\footnotetext{
* Corresponding author

Email addresses: elena.zattoni@unibo.it (Elena Zattoni), perdon@univpm.it (Anna Maria Perdon), gconte@univpm.it (Giuseppe Conte).
}

brid systems of the same class in (Carnevale et al., 2012a, 2013, 2016).

The works by Carnevale et al. (2012a, 2013, 2016) - which, referring to the multivariable case, are closer to this one give a necessary and sufficient condition for the existence of a solution to the considered problem in terms of solvability of a set of differential linear matrix equations. This result is derived by elaborating further on the regulator equations that originally characterized solvability of the output regulation problem for linear time-invariant systems (Francis, 1977). In principle, the hybrid regulator can be obtained by solving the so-called hybrid regulator equations (Carnevale et al., 2016, Section III). However, as acknowledged by the same authors (Carnevale et al., 2016, Remark 2), this method presents serious difficulties due to the infinite number of constraints implied in such equations. For this reason, the analysis is deepened so as to define a viable, valid in general, synthesis procedure, based on the solution of two finitedimensional Francis equations, which are algebraic matrix equations (Carnevale et al., 2016, Section IV-C).

In this work, the output regulation problem for multivariable hybrid systems with periodic state jumps is considered from a different perspective. Namely, new solvability conditions are derived by employing the methodologies of the geometric approach (Wonham, 1985; Basile and Marro, 1992). More specifically, the results presented herein are obtained by exploiting the geometric interpretation of the output regulation problem that was first developed by Marro (1996) and that has lately inspired the solution of the same problem for more complex dynamical systems, such as linear parameter varying systems (Zattoni, 2008), linear time-delay systems (Conte et al., 2012), and linear switching systems (Zattoni et al., 2013). This methodology requires that some fundamental concepts of the geometric approach (e.g., in- 
variance and controlled invariance) be generalized to hybrid systems with state jumps. Indeed, some of these notions have proven to be instrumental in analyzing the structure of the considered hybrid systems in (Medina, 2007; Medina and Lawrence, 2009) and, to some extent, in (Carnevale et al., 2014a). However, in this work, as in (Perdon et al., 2015, 2016), structural notions are used in combination with qualitative notions such as stability, thus allowing all the aspects of the problem to be handled in the geometric framework. A main contribution of this work is establishing a new necessary and sufficient condition for problem solvability in strict geometric terms (Theorem 1). The geometric necessary and sufficient condition is perfectly consistent with the necessary and sufficient condition based on the hybrid regulator equations of (Carnevale et al., 2016, Proposition 3), as will be explained in Remark 1. Meanwhile, the geometric condition has the merit of giving insight into the way the overall regulated system works, since, in particular, it points out the subspace of the admissible state motions. However, the necessary and sufficient geometric condition cannot be directly used as a design tool, since, referring to the overall compensated system, it involves the feedback regulator. Nonetheless, the geometric condition can be exploited to derive a more specific, sufficient solvability condition which provides particularly straightforward synthesis tools (although not applicable to the whole generality of solvable problems).

Hence, the subsequent contribution of this work is a geometric sufficient condition for problem solvability, solely involving the problem data (Theorem 2). In fact, such sufficient condition is centred on the output-difference connection between the plant and the exogenous system (the socalled hybrid extended system) and requires the existence of a subspace with the property of being both controlled invariant for the flow dynamics and invariant for the jump dynamics (in addition to that of being contained in the kernel of the output map). Indeed, such sufficient condition may be rather conservative, a main reason being that it respectively demands controlled invariance and invariance under the linear maps of the flow and jump dynamics (i.e., hybrid controlled invariance) instead of a combination of the two. On the positive side, since such condition disregards the time period between two consecutive state jumps, it ensures the existence of a solution for any finite time period. Further, under such condition, the compensator synthesis is extremely simple. In fact, as will be shown in the proof of the theorem, the synthesis procedure amounts to the computation of a stabilizing friend for the resolving hybrid controlled invariant subspace and of a stabilizing output injection for the dynamics of the hybrid extended system.

In order to shed light on the conflict between conservativeness and constructiveness of the considered conditions, another sufficient condition for problem solvability is established (Theorem 3). Actually, Theorem 3 is focused on the dynamics obtained as the combination over one period of the flow and jump dynamics of the hybrid output-difference system and, as such, has a broader scope compared to Theorem 2. Namely, the condition of Theorem 3 implies that of Theorem 2, while the converse is not true in general, as will be made clear in Remark 5. Nevertheless, it is worth noting that the flow dynamics combined with the jump dynamics in the statement of Theorem 3 is assumed to be compensated by state feedback, so as to take into account the available control input in the way compatible with the compensation scheme considered in the general necessary and sufficient condition. Thus, the interplay between such unknown state feedback and the unknown controlled invariant subspace, which has the role of resolving subspace, makes the condition of Theorem 3 difficult to ascertain and nonconstructive. As mentioned above, the compensator synthesis performed according to the proof of Theorem 2 presupposes that the resolving hybrid controlled invariant subspace be known. However, the geometric sufficient condition does not contain any hint on how to compute such subspace. Hence, in order to provide a complete synthesis tool for the whole set of problems whose solvability is ensured by Theorem 2, a necessary and sufficient constructive condition for a hybrid controlled invariant subspace to satisfy the requisites of Theorem 2 is established in Theorem 4.

The paper is organized as follows. In Section 2, the output regulation problem for multivariable hybrid linear systems with periodic state jumps is presented. In Section 3, a necessary and sufficient condition for problem solvability, referring to the overall compensated hybrid system, is stated in geometric terms. A geometric sufficient condition, focused on the hybrid extended system, is proven in Section 4. In the same section, a more extensive, yet nonconstructive, sufficient condition is also discussed. A necessary and sufficient condition for the existence of a subspace fulfilling the requirements of the constructive sufficient condition is shown in Section 5. A numerical example illustrating how to implement the devised synthesis procedure is worked out in Section 6. Section 7 contains the conclusions. The appendix discusses some results on the stabilization of a hybrid dynamics via a state feedback or via an output injection, each one acting on the flow dynamics only.

Notation: The symbols $\mathbb{Z}, \mathbb{Z}_{0}^{+}, \mathbb{Z}^{+}, \mathbb{R}, \mathbb{R}_{0}^{+}, \mathbb{R}^{+}$, and $\mathbb{C}$ stand for the sets of integer numbers, nonnegative integer numbers, positive integer numbers, real numbers, nonnegative real numbers, positive real numbers, and complex numbers, respectively. The symbol $i$ stands for the imaginary unit and, given a complex number $\lambda=\lambda_{a}+i \lambda_{b},|\lambda|$ denotes its modulus and $\operatorname{Arg}(\lambda)$ its argument. Matrices and linear maps are denoted by slanted capital letters, like $A$. The image, the kernel, the inverse, and the transpose of $A$ are denoted by $\operatorname{Im} A$, $\operatorname{Ker} A, A^{-1}$, and $A^{\top}$, respectively. Vector spaces and subspaces are denoted by calligraphic letters, like $\mathcal{V}$. The notation $\mathcal{W} / \mathcal{V}$ stands for the quotient space of a subspace $\mathcal{W} \subseteq \mathcal{X}$ over a subspace $\mathcal{V} \subseteq \mathcal{W}$. The expression $\mathcal{V} \oplus \mathcal{W}=\mathcal{X}$ stands for $\mathcal{V}+\mathcal{W}=\mathcal{X}$ and $\mathcal{V} \cap \mathcal{W}=\{0\}$. The symbol $\left.A\right|_{\mathcal{J}}$ denotes the restriction of a linear map $A$ to an $A$-invariant subspace $\mathcal{J}$, while $\left.A\right|_{\mathcal{X} / \mathcal{J}}$ denotes the map induced by $A$ on the quotient space $\mathcal{X} / \mathcal{J}$. The symbol $\|x\|$, where $x \in \mathbb{R}^{n}$, denotes the 2-norm of $x$, while $\|A\|$, where $A \in \mathbb{R}^{m \times n}$, denotes the norm induced on $A$ by the vector 2-norm: i.e., $\|A\|=\sup _{x \in \mathbb{R}^{n}, x \neq 0}(\|A x\| /\|x\|)$. Moreover, $\|A\|_{\mathscr{F}}$ denotes the Frobenius norm of $A$ (i.e., the square root of the sum of the squares of all entries). The symbols $I_{n}$ and $0_{m \times n}$ denote an identity matrix of dimension $n$ and an $(m \times n)$-zero matrix (subscripts are omitted when the size is clear from the context). The symbol $\operatorname{diag}\left\{d_{1}, \ldots, d_{n}\right\}$ denotes a diagonal matrix with the elements $d_{1}, \ldots, d_{n}$ on the main diagonal. The notation $\left[t_{0} t_{1}\right)$, with $t_{0}, t_{1} \in \mathbb{R}$, stands for the real right-open interval delimited by $t_{0}$ and $t_{1}$. 


\section{The Output Regulation Problem for Hybrid Systems with Periodic State Jumps}

The aim of this section is to introduce the output regulation problem for hybrid systems with a continuous-time linear dynamics subject to periodic state jumps.

The given plant is the hybrid linear system

$$
\Sigma_{P} \equiv\left\{\begin{aligned}
& \dot{x}_{P}(t)= A_{P} x_{P}(t)+B_{P} u(t), \text { with } \\
& t \in[k \tau,(k+1) \tau), k \in \mathbb{Z}_{0}^{+}, \\
& x_{P}(t)=J_{P} x_{P}^{-}(t), \text { with } t=k \tau, k \in \mathbb{Z}^{+}, \\
& y(t)=C_{P} x_{P}(t),
\end{aligned}\right.
$$

where $t \in \mathbb{R}_{0}^{+}$is the time variable, $\tau \in \mathbb{R}^{+}$is a finite time constant, $x_{P} \in \mathcal{X}_{P}=\mathbb{R}^{n_{P}}$ is the state, $u \in \mathcal{U}=\mathbb{R}^{p}$ is the control input, and $y \in \mathcal{Y}=\mathbb{R}^{q}$ is the regulated output, with $p, q \leq n_{P} . A_{P}, B_{P}, J_{P}$, and $C_{P}$ are constant real matrices. $B_{P}$ and $C_{P}$ are assumed to be full-rank. The initial state is $x_{P 0}$. The set of the admissible input functions is the set of all piecewise-continuous functions with values in $\mathbb{R}^{p}$. According to (1), the state trajectory $x_{P}(t)$ on the time interval $[k \tau,(k+1) \tau)$, with $k \in \mathbb{Z}_{0}^{+}$, is the solution of the system of differential equations with the initial condition $x_{P}(k \tau)$ and the input function $u(t), t \in[k \tau,(k+1) \tau)$, while the state $x_{P}(k \tau)$, with $k \in \mathbb{Z}^{+}$, is the image by $J_{P}$ of $x_{P}^{-}(k \tau)=\lim _{t \rightarrow k \tau^{-}} x_{P}(t)$. It is worth mentioning that a hybrid linear system like $\Sigma_{P}$ is globally asymptotically stable if and only if the state transition matrix between two consecutive jump time instants - i.e., $J_{P} e^{A_{P} \tau}-$ is Schur stable (i.e., all the eigenvalues lie inside the open unit disc of the complex plane). It is also worth noting that, in the case of hybrid linear systems subject to time-driven, periodic state jumps, the notation in (1) is equivalent to that established in (Goebel et al., 2009). However, since this notation does not include the differential equation of the clock variable (redundant in this case), it is better suited to the scope of this work and will be adopted henceforth.

The given exogenous system is the hybrid linear system

$$
\Sigma_{E} \equiv\left\{\begin{aligned}
& \dot{x}_{E}(t)= A_{E} x_{E}(t), \text { with } \\
& t \in[k \tau,(k+1) \tau), k \in \mathbb{Z}_{0}^{+}, \\
& x_{E}(t)=J_{E} x_{E}^{-}(t), \text { with } t=k \tau, k \in \mathbb{Z}^{+}, \\
& r(t)=C_{E} x_{E}(t),
\end{aligned}\right.
$$

where $x_{E} \in \mathbb{R}^{n_{E}}$ is the state and $r \in \mathbb{R}^{q}$ is the reference, with $q \leq n_{E} . A_{E}, J_{E}$, and $C_{E}$ are constant real matrices. $C_{E}$ is assumed to be full-rank. The initial state is $x_{E}$. Moreover, in order to leave apart from consideration the trivial case where the reference goes to zero as the time approaches infinity, it is assumed that the state transition matrix between two consecutive jumps, $J_{E} e^{A_{E} \tau}$, is Schur antistable (i.e., all the eigenvalues lie outside the open unit disc).

The to-be-designed feedback regulator is the hybrid linear system

$$
\Sigma_{R} \equiv\left\{\begin{aligned}
& \dot{x}_{R}(t)= A_{R} x_{R}(t)+B_{R} e(t), \text { with } \\
& t \in[k \tau,(k+1) \tau), k \in \mathbb{Z}_{0}^{+}, \\
& x_{R}(t)= J_{R} x_{R}^{-}(t), \text { with } t=k \tau, k \in \mathbb{Z}^{+}, \\
& u(t)= C_{R} x_{R}(t),
\end{aligned}\right.
$$

where $x_{R} \in \mathbb{R}^{n_{R}}$ is the state and $e \in \mathbb{R}^{q}$, defined as $e(t)=$ $r(t)-y(t)$, is the regulation error. The initial state is $x_{R 0}$. In order to state the output regulation problem, the closedloop connection of the plant and the feedback regulator, with the additional input $r(t)$, is defined as

$$
\Sigma_{L} \equiv\left\{\begin{aligned}
\dot{x}_{L}(t)= & A_{L} x_{L}(t)+B_{L} r(t), \text { with } \\
t \in[k \tau,(k+1) \tau), k \in \mathbb{Z}_{0}^{+} & \\
x_{L}(t)= & J_{L} x_{L}^{-}(t), \text { with } t=k \tau, k \in \mathbb{Z}^{+} \\
e(t)= & C_{L} x_{L}(t)+r(t),
\end{aligned}\right.
$$

where

$$
\begin{aligned}
A_{L} & =\left[\begin{array}{cc}
A_{P} & B_{P} C_{R} \\
-B_{R} C_{P} & A_{R}
\end{array}\right], \quad B_{L}=\left[\begin{array}{c}
0 \\
B_{R}
\end{array}\right], \\
J_{L} & =\left[\begin{array}{cc}
J_{P} & 0 \\
0 & J_{R}
\end{array}\right], \\
C_{L} & =\left[\begin{array}{ll}
-C_{P} & 0
\end{array}\right] .
\end{aligned}
$$

Problem 1 (Output Regulation with Global Asymptotic Stability of the Closed-loop Dynamics) Given the plant $\Sigma_{P}$ and the exogenous system $\Sigma_{E}$, find a feedback regulator $\Sigma_{R}$ such that the system $\Sigma_{L}$ satisfies the following requirements: $\mathcal{R} 1$. (global asymptotic stability of the closed-loop dynamics) the state transition matrix between two consecutive jump time instants, $J_{L} e^{A_{L} \tau}$, is Schur stable;

$\mathcal{R} 2$. (asymptotic tracking of the reference) $\lim _{t \rightarrow \infty} e(t)=$ 0 , for all $x_{P 0}, x_{R 0}$, and $r(t)$ generated by $\Sigma_{E}$.

\section{A Geometric Necessary and Sufficient Condition}

In this section, a necessary and sufficient condition for the existence of a solution to Problem 1 is derived by exploiting the geometric properties of the so-called hybrid autonomous system. The hybrid autonomous system $\hat{\Sigma}$ is defined as the connection of the closed-loop system $\Sigma_{L}$ and the exogenous system $\Sigma_{E}$ described below:

$\hat{\Sigma} \equiv\left\{\begin{array}{l}\dot{\hat{x}}(t)=\hat{A} \hat{x}(t), \text { with } t \in[k \tau,(k+1) \tau), k \in \mathbb{Z}_{0}^{+}, \\ \hat{x}(t)=\hat{J} \hat{x}^{-}(t), \text { with } t=k \tau, k \in \mathbb{Z}^{+}, \\ e(t)=\hat{E} \hat{x}(t),\end{array}\right.$

where

$$
\begin{aligned}
& \hat{A}=\left[\begin{array}{cc}
A_{L} & B_{L} C_{E} \\
0 & A_{E}
\end{array}\right], \\
& \hat{J}=\left[\begin{array}{cc}
J_{L} & 0 \\
0 & J_{E}
\end{array}\right], \\
& \hat{E}=\left[\begin{array}{ll}
C_{L} & C_{E}
\end{array}\right] .
\end{aligned}
$$

The state space $\mathbb{R}^{\hat{n}}=\mathbb{R}^{n_{L}+n_{E}}$ of $\hat{\Sigma}$ is denoted by $\hat{\mathcal{X}}$; the kernel of $\hat{E}$ is denoted by $\hat{\mathcal{E}}$; the unobservable subspace of $(\hat{A}, \hat{E})$ is denoted by $\hat{\mathcal{O}}$ or, equivalently, by $\max \mathcal{J}(\hat{A}, \hat{\mathcal{E}})$ - i.e., the maximal $\hat{A}$-invariant subspace contained in $\hat{\mathcal{E}}$. Moreover, the maximal $\hat{J} e^{\hat{A} \tau}$-invariant subspace contained in $\hat{\mathcal{O}}$ - namely, $\max \mathcal{J}\left(\hat{J} e^{\hat{A} \tau}, \hat{\mathcal{O}}\right)-$ is denoted by $\hat{\mathcal{Q}}$. It 
is worth noting that any state trajectory of $\hat{\Sigma}$ starting from a state in $\hat{\mathcal{Q}}$ gives zero output for all $t \in \mathbb{R}_{0}^{+}$.

In order to state the necessary and sufficient condition, the subspace $\hat{\mathcal{P}} \subseteq \hat{\mathcal{X}}$ is introduced through the following definition and characterized by the subsequent property. Namely,

$$
\hat{\mathcal{P}}=\operatorname{Im} \hat{P}=\operatorname{Im}\left[\begin{array}{ll}
I & 0
\end{array}\right]^{\top},
$$

where the partition of $\hat{P}$ is consistent with that of (5)-(7).

Property 1 The subspace $\hat{\mathcal{P}}$ is invariant under the linear map $\hat{J} e^{\hat{A} \tau}$. The restriction of $\hat{J} e^{\hat{A} \tau}$ to $\hat{\mathcal{P}}$ and the linear map induced by $\hat{J} e^{\hat{A} \tau}$ on the quotient space $\hat{\mathcal{X}} / \hat{\mathcal{P}}$ respectively are

$$
\begin{aligned}
& \left.\hat{J} e^{\hat{A} \tau}\right|_{\hat{\mathcal{P}}}=J_{L} e^{A_{L} \tau}, \\
& \left.\hat{J} e^{\hat{A} \tau}\right|_{\hat{\mathcal{X}} / \hat{\mathcal{P}}}=J_{E} e^{A_{E} \tau} .
\end{aligned}
$$

Proof. Note that, in light of (5) and (6),

$$
\hat{J} e^{\hat{A} \tau}=\left[\begin{array}{cc}
J_{L} e^{A_{L} \tau} & * \\
0 & J_{E} e^{A_{E} \tau}
\end{array}\right],
$$

where $*$ stands for a matrix block of no interest to this discussion. Hence, the upper block-triangular structure of (11) proves invariance of $\hat{\mathcal{P}}$, while the matrix blocks on the main diagonal respectively prove (9) and (10).

In view of the proof of the following theorem, it is worth noting that $\Sigma_{L}$ is a subsystem of $\hat{\Sigma}$ and $\hat{\mathcal{P}}$ is its state space.

Theorem 1 Problem 1 has a solution if and only if there exists a subspace $\hat{\mathcal{V}} \subseteq \hat{\mathcal{X}}$, invariant under the linear map $\hat{J} e^{\hat{A} \tau}$, such that

C $1 . \hat{\mathcal{V}} \oplus \hat{\mathcal{P}}=\hat{\mathcal{X}}$

$\mathcal{C} 2 . \hat{\mathcal{V}} \subseteq \hat{\mathcal{Q}}$;

C 3. $\left.\hat{J} e^{\hat{A} \tau}\right|_{\hat{\mathcal{X}} / \hat{\mathcal{V}}}$ is Schur stable.

Proof. Only if. Let Problem 1 have a solution. Let $\hat{\mathcal{V}}$ be the sum of the generalized eigenspaces of $\hat{J} e^{\hat{A} \tau}$ associated to the eigenvalues lying outside the open unit disc of the complex plane. Hence, the subspace $\hat{\mathcal{V}}$ is invariant under the linear map $\hat{J} e^{\hat{A} \tau}$ by definition. Moreover, for the same reason, $\left.\hat{J} e^{\hat{A} \tau}\right|_{\hat{\mathcal{V}}}$ is Schur antistable, while $\left.\hat{J} e^{\hat{A} \tau}\right|_{\hat{\mathcal{X}} / \hat{\mathcal{V}}}$ is Schur stable - i.e., Condition $\mathcal{C} 3$ holds. On the other hand, Requirement $\mathcal{R} 1$ of Problem 1 , which is met by assumption, and (9) in Property 1 imply that $\left.\hat{J} e^{\hat{A} \tau}\right|_{\hat{\mathcal{P}}}$ is Schur stable. Meanwhile, (10) in Property 1 and the assumption that $J_{E} e^{A_{E} \tau}$ is Schur antistable, imply that $\left.\hat{J} e^{\hat{A} \tau}\right|_{\hat{\mathcal{X}} / \hat{\mathcal{P}}}$ is Schur antistable. Therefore, the comparison between the spectra of the restriction of the linear map $\hat{J} e^{\hat{A} \tau}$ to $\hat{\mathcal{V}}$ and $\hat{\mathcal{P}}$ and the spectra of the linear maps respectively induced by $\hat{J} e^{\hat{A} \tau}$ on $\hat{\mathcal{X}} / \hat{\mathcal{V}}$ and $\hat{\mathcal{X}} / \hat{\mathcal{P}}$ shows that Condition $\mathcal{C} 1$ holds. In order to show that Condition $\mathcal{C} 2$ is fulfilled, first note that $\hat{\mathcal{Q}}$ is defined as the maximal $\hat{J} e^{\hat{A} \tau}$-invariant subspace contained in $\hat{\mathcal{O}}$ and that, as was proven above, $\hat{\mathcal{V}}$ is a $\hat{J} e^{\hat{A} \tau}$-invariant subspace. Hence, showing that $\hat{\mathcal{V}} \subseteq \hat{\mathcal{Q}}$ reduces to showing that $\hat{\mathcal{V}} \subseteq \hat{\mathcal{O}}$. To this aim, note that any vector $\hat{x} \in \hat{\mathcal{V}}$ can be written as

$$
\hat{x}=\sum_{i=1}^{h} \sum_{j=1}^{j_{i}} c_{i j} v_{i j}+\sum_{i^{\prime}=1}^{h^{\prime}} \sum_{j^{\prime}=1}^{j_{i^{\prime}}}\left(\alpha_{i^{\prime} j^{\prime}} w_{i^{\prime} j^{\prime}}+\bar{\alpha}_{i^{\prime} j^{\prime}} \bar{w}_{i^{\prime} j^{\prime}}\right),
$$

where $h$ is the number of Jordan blocks in the Jordan form of $\hat{J} e^{\hat{A} \tau}$ corresponding to real eigenvalues that lie outside the open unit disc of the complex plane and $j_{i}$ is the dimension of the $i$-th Jordan block; similarly, $2 h^{\prime}$ is the number of Jordan blocks corresponding to complex eigenvalues that lie outside the open unit disc of the complex plane and $j_{i^{\prime}}$ is the dimension of the $i^{\prime}$-th Jordan block; the coefficients $c_{i j}$ are real numbers and the vectors $v_{i j}$ are real vectors that, for $j=1, \ldots, j_{i}$, form a Jordan chain of generalized eigenvectors associated to the $i$-th Jordan block; the coefficients $\alpha_{i^{\prime} j^{\prime}}, \bar{\alpha}_{i^{\prime} j^{\prime}}$ are conjugate complex numbers and the vectors $w_{i^{\prime} j^{\prime}}, \bar{w}_{i^{\prime} j^{\prime}}$ are conjugate complex vectors that, for $j^{\prime}=1, \ldots, j_{i^{\prime}}$, form two Jordan chains of generalized eigenvectors respectively associated to the $i^{\prime}$-th and to the $\left(i^{\prime}+h^{\prime}\right)$-th Jordan blocks - it is assumed that the Jordan blocks are ordered in such a way that the $i^{\prime}$-th and the $\left(i^{\prime}+h^{\prime}\right)$-th blocks correspond to a pair of conjugate complex eigenvalues. Let $\lambda_{i}$, denote the real eigenvalue associated to $i$-th Jordan block, with $i=1, \ldots, h$. Then, recall that the corresponding real generalized eigenvectors satisfy $\hat{J} e^{\hat{A} \tau} v_{i 1}=\lambda_{i} v_{i 1}$ and $\left(\hat{J} e^{\hat{A} \tau}-\lambda_{i} I\right) v_{i j}=v_{i(j-1)}$ with $i=1, \ldots, h$ and $j=2, \ldots, j_{i}$. Moreover, let $\lambda_{i^{\prime}}$ and $\bar{\lambda}_{i^{\prime}}$ denote the pair of conjugate complex eigenvalues respectively associated to the $i^{\prime}$-th and $\left(i^{\prime}+h^{\prime}\right)$-th Jordan blocks. Then, the corresponding conjugate complex generalized eigenvectors satisfy $\hat{J} e^{\hat{A} \tau} w_{i^{\prime} 1}=\lambda_{i^{\prime}} w_{i^{\prime} 1}$, $\left(\hat{J} e^{\hat{A} \tau}-\lambda_{i^{\prime}} I\right) w_{i^{\prime} j^{\prime}}=w_{i^{\prime}\left(j^{\prime}-1\right)}, \quad \hat{J} e^{\hat{A} \tau} \bar{w}_{i^{\prime} 1}=\bar{\lambda}_{i^{\prime}} \bar{w}_{i^{\prime} 1}$, and $\left(\hat{J} e^{\hat{A} \tau}-\bar{\lambda}_{i^{\prime}} I\right) \bar{w}_{i^{\prime} j^{\prime}}=\bar{w}_{i^{\prime}\left(j^{\prime}-1\right)}$, with $i^{\prime}=1, \ldots, h^{\prime}$ and $j^{\prime}=2, \ldots, j_{i^{\prime}}$. Consider the Jordan chain of real generalized eigenvectors corresponding to the $i$-th Jordan block and to the real eigenvalue $\lambda_{i}$ and, by dropping the index $i$ to simplify the notation, represent it by $\left\{v_{1}, \ldots, v_{j_{i}}\right\}$. Let $\hat{x}(t)$, with $t \in \mathbb{R}_{0}^{+}$, denote the state trajectory of $\hat{\Sigma}$ starting from $\hat{x}(0) \in \hat{\mathcal{V}}$ and consider the corresponding output, $e(t)=\hat{E} \hat{x}(t)$. Let $\hat{x}(0)=v_{1}$. Then, with $k \tau \leq t<(k+1) \tau$, the output is given by $e(t)=\hat{E} e^{\hat{A}(t-k \tau)}\left(\hat{J} e^{\hat{A} \tau}\right)^{k} v_{1}=\lambda^{k} \hat{E} e^{\hat{A}(t-k \tau)} v_{1}$. Since $|\lambda|>1$, Requirement $\mathcal{R} 2$ of Problem 1 , which is satisfied by assumption, implies that $\hat{E} e^{\hat{A}(t-k \tau)} v_{1}=\hat{E} e^{\hat{A} t^{\prime}} v_{1}=0$ for all $t^{\prime}=(t-k \tau) \in[0, \tau)$, which, in turn, implies that $v_{1}$ belongs to $\hat{\mathcal{O}}$. Hence, let $\hat{x}(0)=v_{2}$. Then, with $k \tau \leq t<(k+1) \tau$, the output is given by $e(t)=\hat{E} e^{\hat{A}(t-k \tau)}\left(\hat{J} e^{\hat{A} \tau}\right)^{k} v_{2}=\hat{E} e^{\hat{A}(t-k \tau)}\left(k \lambda^{k-1} v_{1}+\right.$ $\left.\lambda^{k} v_{2}\right)$, which implies that $e(t)=\lambda^{k} \hat{E} e^{\hat{A}(t-k \tau)} v_{2}$, by the previous result. As before, it follows that $v_{2}$ belongs to $\hat{\mathcal{O}}$ and, in the same way, it follows that $v_{j}$ belongs to $\hat{\mathcal{O}}$ for all the remaining values of $j$. Hence, the conclusion is that the real eigenvector $v_{i j}$ belongs to $\hat{\mathcal{O}}$ for any $i j$. Next, consider the Jordan chain of complex generalized eigenvectors corresponding to the $i^{\prime}$-th Jordan block and to the complex eigenvalue $\lambda_{i^{\prime}}$ and, by dropping the index $i^{\prime}$ to simplify the notation, represent it by $\left\{w_{1}, \ldots, w_{j_{i^{\prime}}}\right\}$. Also consider the Jordan chain of conjugate complex generalized eigenvectors corresponding to the $\left(i^{\prime}+h^{\prime}\right)$-th Jordan block and to the conjugate complex eigenvalue $\bar{\lambda}_{i^{\prime}}$, and, similarly, represent it by $\left\{\bar{w}_{1}, \ldots, \bar{w}_{j_{i^{\prime}}}\right\}$. Let $\hat{x}(0)=\alpha_{1} w_{1}+\bar{\alpha}_{1} \bar{w}_{1}$. 
Then, with $k \tau \leq t<(k+1) \tau$, the output is given by $e(t)=\hat{E} e^{\hat{A}(t-k \tau)}\left(\hat{J} e^{\hat{A} \tau}\right)^{k} \hat{x}(0)=\hat{E} e^{\hat{A}(t-k \tau)}\left(\alpha_{1} \lambda^{k} w_{1}+\right.$ $\left.\bar{\alpha}_{1} \bar{\lambda}^{k} \bar{w}_{1}\right)$. With reference to the notation $\lambda=|\lambda| e^{i \operatorname{Arg}(\lambda)}$, where $i$ denotes the imaginary unit, let us first consider the case where $\operatorname{Arg}(\lambda) / 2 \pi$ is a rational number: i.e., let $\operatorname{Arg}(\lambda) / 2 \pi=l^{\prime} / l$, with $l, l^{\prime}$ integer numbers (and $l$ positive). Hence, $\lambda^{k l}=|\lambda|^{k l} e^{i k l \operatorname{Arg}(\lambda)}=|\lambda|^{k l} e^{i 2 k l^{\prime} \pi}=|\lambda|^{k l}$ and $\bar{\lambda}^{k l}=|\bar{\lambda}|^{k l} e^{i k l \operatorname{Arg}(\bar{\lambda})}=|\lambda|^{k l} e^{-i 2 k l^{\prime} \pi}=|\lambda|^{k l}$. Therefore, with $k l \tau \leq t<(k l+1) \tau$, the output is $e(t)=|\lambda|^{k l} \hat{E} e^{\hat{A}(t-k l \tau)}\left(\alpha_{1} w_{1}+\bar{\alpha}_{1} \bar{w}_{1}\right)$. Since $|\lambda|>1$, Requirement $\mathcal{R} 2$ of Problem 1 (met by assumption) implies that $\hat{E} e^{\hat{A}(t-k l \tau)}\left(\alpha_{1} w_{1}+\bar{\alpha}_{1} \bar{w}_{1}\right)=\hat{E} e^{\hat{A} t^{\prime}}\left(\alpha_{1} w_{1}+\right.$ $\left.\bar{\alpha}_{1} \bar{w}_{1}\right)=0$ for all $t^{\prime}=(t-k l \tau) \in[0, \tau)$ and, therefore, $\alpha_{1} w_{1}+\bar{\alpha}_{1} \bar{w}_{1}$ belongs to $\hat{\mathcal{O}}$ for any $\alpha_{1} \in \mathbb{C}$. Otherwise, if $\operatorname{Arg}(\lambda) / 2 \pi$ is not a rational number, it is possible to find a sequence of integers $\left\{k_{n} ; n \in \mathbb{Z}^{+}\right\}$, with $\lim _{n \rightarrow+\infty} k_{n}=+\infty$, such that, given a neighborhood of radius $\epsilon$ of $\hat{x}_{0}=\alpha_{1} w_{1}+\bar{\alpha}_{1} \bar{w}_{1}$, say $S\left(\hat{x}_{0}, \epsilon\right)$, the (real) points $\hat{x}_{n}=\alpha_{1} e^{i k_{n} \operatorname{Arg}(\lambda)} w_{1}+\bar{\alpha}_{1} e^{i k_{n} \operatorname{Arg}(\bar{\lambda})} \bar{w}_{1}$ belong to $S\left(\hat{x}_{0}, \epsilon\right)$ for any $n \in \mathbb{Z}^{+}$. In particular, since for any $t^{\prime} \in[0, \tau)$ the linear map $\hat{E} e^{\hat{A} t^{\prime}}: \hat{\mathcal{X}} \rightarrow \mathcal{Y}$ is continuous, it is possible to choose $\epsilon$ in such a way that $\left\|\hat{E} e^{\hat{A} t^{\prime}} \hat{x}_{n}\right\| \geq\left\|\hat{E} e^{\hat{A} t^{\prime}} \hat{x}_{0}\right\| / 2$. Therefore, with $k_{n} \tau \leq t<\left(k_{n}+1\right) \tau$, the output is given by $e(t)=$ $|\lambda|^{k_{n}} \hat{E} e^{\hat{A}\left(t-k_{n} \tau\right)}\left(\alpha_{1} e^{i k_{n} \operatorname{Arg}(\lambda)} w_{1}+\bar{\alpha}_{1} e^{i k_{n} \operatorname{Arg}(\bar{\lambda})} \bar{w}_{1}\right)=$ $|\lambda|^{k_{n}} \hat{E} e^{\hat{A} t^{\prime}} \hat{x}_{n}$, where $t^{\prime}=\left(t-k_{n} \tau\right) \in[0, \tau)$, and $\|e(t)\| \geq$ $|\lambda|^{k_{n}}\left\|\hat{E} e^{\hat{A} t^{\prime}} \hat{x}_{0}\right\| / 2$. Since $|\lambda|>1$, Requirement $\mathcal{R} 2 \mathrm{im}-$ plies that $\hat{E} e^{\hat{A} t^{\prime}} \hat{x}_{0}=\hat{E} e^{\hat{A} t^{\prime}}\left(\alpha_{1} w_{1}+\bar{\alpha}_{1} \bar{w}_{1}\right)=0$ for all $t^{\prime}=(t-k \tau) \in[0, \tau)$ and any $\alpha_{1} \in \mathbb{C}$ and, therefore, also in this case, $\alpha_{1} w_{1}+\bar{\alpha}_{1} \bar{w}_{1}$ belongs to $\hat{\mathcal{O}}$ for any $\alpha_{1} \in \mathbb{C}$.

If. Let the subspace $\hat{\mathcal{V}} \subseteq \hat{\mathcal{X}}$ be invariant under the linear map $\hat{J} e^{\hat{A} \tau}$ and satisfy Conditions $\mathcal{C} 1-\mathcal{C} 3$. Then, Conditions $\mathcal{C} 1$ and $\mathcal{C} 3$, in light of Property 1 , imply that Requirement $\mathcal{R} 1$ of Problem 1 is met. In fact, the spectra of $\left.\hat{J} e^{\hat{A} \tau}\right|_{\hat{\mathcal{X}} / \hat{\mathcal{V}}}$ and $\left.\hat{J} e^{\hat{A} \tau}\right|_{\hat{\mathcal{P}}}$ are equal. Moreover, Conditions $\mathcal{C} 1, \mathcal{C} 2$, and $\mathcal{C} 3$, in light of Property 1 , imply that Requirement $\mathcal{R} 2$ of Problem 1 is also met. In fact, by Condition $\mathcal{C} 1$, any state $\hat{x}_{0} \in \hat{\mathcal{X}}$ can be written, in a unique way, as $\hat{x}_{0}=\hat{x}_{1}+\hat{x}_{2}$ with $\hat{x}_{1} \in \hat{\mathcal{P}}$ and $\hat{x}_{2} \in \hat{\mathcal{V}}$. Thus, the corresponding trajectory $\hat{x}(t)$ of $\hat{\Sigma}$, with $t \in[k \tau,(k+1) \tau)$, can be written as $\hat{x}(t)=e^{\hat{A}(t-k \tau)}\left(\hat{J} e^{\hat{A} \tau}\right)^{k} \hat{x}_{0}=e^{\hat{A}(t-k \tau)}\left(\hat{J} e^{\hat{A} \tau}\right)^{k}\left(\hat{x}_{1}+\hat{x}_{2}\right)$ $=e^{\hat{A}(t-k \tau)}\left(\left(\hat{J} e^{\hat{A} \tau}\right)^{k} \hat{x}_{1}+\left(\hat{J} e^{\hat{A} \tau}\right)^{k} \hat{x}_{2}\right)$,

where

$\left(\hat{J} e^{\hat{A} \tau}\right)^{k} \hat{x}_{1}$ belongs to $\hat{\mathcal{P}}$ and $\left(\hat{J} e^{\hat{A} \tau}\right)^{k} \hat{x}_{2}$ belongs to $\hat{\mathcal{V}}$, because $\hat{\mathcal{P}}$ and $\hat{\mathcal{V}}$ are $\hat{J} e^{\hat{A} \tau}$-invariant. Then, by Condition $\mathcal{C} 2, \quad e(t)=\hat{E} \hat{x}(t)=\hat{E} e^{\hat{A}(t-k \tau)}\left(\hat{J} e^{\hat{A} \tau}\right)^{k} \hat{x}_{1} \quad$ and $\lim _{t \rightarrow+\infty}\|\hat{E} \hat{x}(t)\| \leq\left(\max _{t^{\prime} \in[0, \tau]}\left\|\hat{E} e^{\hat{A} t^{\prime}}\right\|\right)\left(\lim _{k \rightarrow \infty}\left\|\left(\hat{J} e^{\hat{A} \tau}\right)^{k} \hat{x}_{1}\right\|\right)$. Since, as noticed above, the spectra of $\left.\hat{J} e^{\hat{A} \tau}\right|_{\hat{\mathcal{X}} / \hat{\mathcal{V}}}$ and $\left.\hat{J} e^{\hat{A} \tau}\right|_{\hat{\mathcal{P}}}$ are equal, it follows that $\lim _{k \rightarrow \infty}\left(\hat{J} e^{\hat{A} \tau}\right)^{k} \hat{x}_{1}=0$ by Condition $\mathcal{C} 3$ and, therefore, $\lim _{t \rightarrow \infty} e(t)=0$ for all $\hat{x}_{0}=\left[\begin{array}{lll}x_{P 0}^{\top} & x_{R 0}^{\top} & x_{E 0}^{\top}\end{array}\right]^{\top} \in \hat{\mathcal{X}}$ or, equivalently, for all $x_{P 0}$, $x_{R 0}$ and $r(t)$ generated by $\Sigma_{E}$.

Remark 1 The result of Theorem 1 is in accordance with the conditions for the solution of Problem 1 given in
(Carnevale et al., 2016). This can be seen by noting that, given a matrix $\hat{V}$ whose columns span $\hat{\mathcal{V}}$, by Condition $\mathcal{C} 1$, one can assume that

$$
\hat{V}=\left[V_{1}^{\top} V_{2}^{\top} I\right]^{\top},
$$

where the partition is consistent with that of (5)-(7), where (2)-(4) have been taken into account. Comparing with the proof of Proposition 3 in (Carnevale et al., 2016), one can see that the steady-state solutions of $\hat{\Sigma}$, with $k \tau \leq t<(k+1) \tau$, can be written as

$\hat{x}(t)=$

$\left[\begin{array}{c}x_{P}(t) \\ x_{R}(t) \\ x_{E}(t)\end{array}\right]=e^{\hat{A}(t-k \tau)}\left[\begin{array}{c}x_{P}(k \tau) \\ x_{R}(k \tau) \\ x_{E}(k \tau)\end{array}\right]=e^{\hat{A}(t-k \tau)}\left[\begin{array}{c}V_{1} \\ V_{2} \\ I\end{array}\right] x_{E}(k \tau)$.

Letting $\sigma=t-k \tau$, recalling the block structure of $\hat{A}$, and noting that $x_{E}(k \tau)=e^{-A_{E} \sigma} x_{E}(t)$, one can write

$\hat{x}(t)=e^{\hat{A} \sigma}\left[\begin{array}{c}V_{1} \\ V_{2} \\ I\end{array}\right] e^{-A_{E} \sigma} x_{E}(t)=\left[\begin{array}{c}V_{1}(\sigma) e^{-A_{E} \sigma} \\ V_{2}(\sigma) e^{-A_{E} \sigma} \\ I\end{array}\right] x_{E}(t)$,

where $V_{1}(\sigma)$ and $V_{2}(\sigma)$ respectively denote the corresponding blocks of rows of the matrix product $e^{\hat{A} \sigma}\left[V_{1}^{\top} V_{2}^{\top} I\right]^{\top}$. Then, it turns out that $V_{1}(\sigma) e^{-A_{E} \sigma}$ and $V_{2}(\sigma) e^{-A_{E} \sigma}$ respectively coincide with the matrices $\Pi(\sigma)$ and $\Sigma(\sigma)$ in (Carnevale et al., 2016). In particular, with the same reasoning as in the quoted proof, it can be shown that these matrices verify the so-called hybrid regulator equations given in formulas (11) of (Carnevale et al., 2016).

In the necessary and sufficient condition for the solution of the output regulation problem expressed in Theorem 1, the matrices of the to-be-designed compensator are implicit in those of the hybrid autonomous system. Hence, the purpose of the following developments is to find conditions under which the regulator matrices can be explicitly determined. In particular, the notion of $\mathscr{H}$-invariant subspace for the hybrid autonomous system and the relation between $\mathscr{H}$-invariance and invariance under the linear map ruling the state transition between two consecutive jump time instants pave the way to the proof of the explicit, sufficient conditions that will be presented in the next section.

Definition $1 A$ subspace $\hat{\mathcal{W}} \subseteq \hat{\mathcal{X}}$ is said to be an $\mathscr{H}$-invariant subspace for the autonomous hybrid system $\hat{\Sigma}$ if $\hat{A} \hat{\mathcal{W}} \subseteq \hat{\mathcal{W}}$ and $\hat{J} \hat{\mathcal{W}} \subseteq \hat{\mathcal{W}}$.

Proposition 1 A subspace $\hat{\mathcal{W}} \subseteq \hat{\mathcal{X}}$, with a basis matrix $\hat{W}$, is an $\mathscr{H}$-invariant subspace for the hybrid autonomous system $\hat{\Sigma}$ if and only if there exist matrices $L_{\hat{A}}$ and $L_{\hat{J}}$, such that

$\hat{A} \hat{W}=\hat{W} L_{\hat{A}}$,

$\hat{J} \hat{W}=\hat{W} L_{\hat{J}}$.

Proposition 1, which characterizes $\hat{\mathcal{W}}$ as an $\mathscr{H}$-invariant subspace for the hybrid autonomous system $\hat{\Sigma}$, expresses the notions of $\hat{A}$-invariance and $\hat{J}$-invariance of $\hat{\mathcal{W}}$ in a coordinate-dependent setting. 
It is noteworthy that, since the notion of $\mathscr{H}$-invariance is merely related to linear maps whose image is in the state space of $\hat{\Sigma}$, it is not affected by the fact that the linear map $\hat{A}$ refers to a continuous-time dynamics, while the linear map $\hat{J}$ does not. In other words, $\mathscr{H}$-invariance of a subspace with respect to a hybrid system is a structural notion and, as such, it encompasses the hybrid nature of the system.

Proposition 2 Let the subspace $\hat{\mathcal{W}} \subseteq \hat{\mathcal{X}}$ be an $\mathscr{H}$-invariant subspace for the hybrid autonomous system $\hat{\Sigma}$. Then, $\hat{\mathcal{W}}$ is invariant under the linear map $\hat{J} e^{\hat{A} \tau}$.

Proof. Let the basis transformation $\hat{T}=\left[\begin{array}{ll}\hat{T}_{1} & \hat{T}_{2}\end{array}\right]$, where $\operatorname{Im} \hat{T}_{1}=\hat{\mathcal{W}}$, be applied in the state space $\hat{\mathcal{X}}$ of $\hat{\Sigma}$. Hence, with respect to the new coordinates,

$\hat{A}^{\prime}=\hat{T}^{-1} \hat{A} \hat{T}=\left[\begin{array}{cc}\hat{A}_{11}^{\prime} & \hat{A}_{12}^{\prime} \\ 0 & \hat{A}_{22}^{\prime}\end{array}\right]$,
$\hat{J}^{\prime}=\hat{T}^{-1} \hat{J} \hat{T}=\left[\begin{array}{cc}\hat{J}_{11}^{\prime} & \hat{J}_{12}^{\prime} \\ 0 & \hat{J}_{22}^{\prime}\end{array}\right]$

where the structural zero matrices in $\hat{A}^{\prime}$ and $\hat{J}^{\prime}$ are respectively due to $\hat{A}$-invariance and $\hat{J}$-invariance of $\hat{\mathcal{W}}$. Then,

$$
\hat{J}^{\prime} e^{\hat{A}^{\prime} \tau}=\left[\begin{array}{cc}
\hat{J}_{11}^{\prime} e^{\hat{A}_{11}^{\prime} \tau} & * \\
0 & \hat{J}_{22}^{\prime} e^{\hat{A}_{22}^{\prime} \tau}
\end{array}\right],
$$

and the upper block-triangular structure proves the thesis.

It is worth noting that, while Proposition 2 states that $\mathscr{H}$-invariance implies invariance with respect to the linear map ruling the state transition between two consecutive jump time instants, the converse is not true in general.

\section{Sufficient Conditions for Problem Solvability}

The object of this section is the discussion of two different sufficient conditions for problem solvability, based on the geometric properties of the so-called hybrid extended system. To this aim, the notion of $\mathscr{H}$-controlled invariant subspace for a hybrid system with control inputs is first revisited and then characterized through a couple of necessary and sufficient conditions.

The hybrid extended system - henceforth denoted by $\Sigma$ is defined as the output-difference connection between the plant $\Sigma_{P}$ and the exogenous system $\Sigma_{E}$ : i.e.,

$$
\Sigma \equiv\left\{\begin{aligned}
& \dot{x}(t)=A x(t)+B u(t), \text { with } \\
& t \in[k \tau,(k+1) \tau), k \in \mathbb{Z}_{0}^{+}, \\
& x(t)=J x^{-}(t), \text { with } t=k \tau, k \in \mathbb{Z}^{+} \\
& e(t)=E x(t),
\end{aligned}\right.
$$

where

$$
A=\left[\begin{array}{cc}
A_{P} & 0 \\
0 & A_{E}
\end{array}\right], \quad B=\left[\begin{array}{c}
B_{P} \\
0
\end{array}\right],
$$

$$
\begin{aligned}
& J=\left[\begin{array}{cc}
J_{P} & 0 \\
0 & J_{E}
\end{array}\right], \\
& E=\left[\begin{array}{ll}
-C_{P} & C_{E}
\end{array}\right] .
\end{aligned}
$$

The state space $\mathbb{R}^{n}=\mathbb{R}^{n_{P}+n_{E}}$ of $\Sigma$ is denoted by $\mathcal{X}$. The subspaces $\operatorname{Im} B$ and $\operatorname{Ker} E$ are respectively denoted by $\mathcal{B}$ and $\mathcal{E}$. For the sake of immediacy, the following statements refer to the hybrid system $\Sigma$. However, it is understood that the peculiar structure of the matrices $A, B, J, E$ shown in (14)-(16) has no influence on these statements.

Definition $2 A$ subspace $\mathcal{V} \subseteq \mathcal{X}$ is said to be an $\mathscr{H}$-controlled invariant subspace for the hybrid system $\Sigma$ if

$$
\begin{aligned}
& A \mathcal{V} \subseteq \mathcal{V}+\mathcal{B} \\
& J \mathcal{V} \subseteq \mathcal{V} .
\end{aligned}
$$

Proposition 3 A subspace $\mathcal{V} \subseteq \mathcal{X}$, with a basis matrix $V$, is an $\mathscr{H}$-controlled invariant subspace for the hybrid system $\Sigma$ if and only if there exist matrices $L_{A}, L_{J}$, and $M$, such that

$$
\begin{aligned}
& A V=V L_{A}+B M, \\
& J V=V L_{J} .
\end{aligned}
$$

Proposition 4 A subspace $\mathcal{V} \subseteq \mathcal{X}$ is an $\mathscr{H}$-controlled invariant subspace for the hybrid system $\Sigma$ if and only if there exists a linear map $F: \mathcal{X} \rightarrow \mathcal{U}$, such that

$$
(A+B F) \mathcal{V} \subseteq \mathcal{V}
$$

holds along with (18).

The first characterization of $\mathcal{V}$ as an $\mathscr{H}$-controlled invariant subspace for the hybrid system $\Sigma$ expresses simultaneous $(A, \mathcal{B})$-controlled invariance and $J$-invariance in a coordinate-dependent setting. The second characterization is shown to be equivalent to the former via the coordinatedependent characterization of $(A+B F)$-invariance: i.e., it ensues from $(A+B F) V=V L_{A}$ by picking $F$ such that $F V=-M$. It is worth noting that Propositions 1 and 3, which respectively characterize an $\mathscr{H}$-invariant subspace and an $\mathscr{H}$-controlled invariant subspace, imply that an $\mathscr{H}$ invariant subspace is an $\mathscr{H}$-controlled invariant subspace for any $\mathcal{B}$. It is also worth noting that, since $\mathscr{H}$-controlled invariance (like $\mathscr{H}$-invariance) only deals with linear maps whose domain belongs to the state space of the hybrid system, it is not affected by the fact that the pair $(A, B)$ refers to a continuous-time dynamics, while the linear map $J$ does not. In order to state the theorem expressing the explicit, sufficient condition for solvability of the output regulation problem — as well as the lemma smoothing the way to its proof - the subspace $\mathcal{P} \subseteq \mathcal{X}$ is introduced through the following definition and the subsequent property. Namely,

$$
\mathcal{P}=\operatorname{Im} P=\operatorname{Im}\left[\begin{array}{ll}
I & 0
\end{array}\right]^{\top},
$$

where the partition of $P$ is consistent with that of (14)-(16).

Property 2 The subspace $\mathcal{P}$ is an $\mathscr{H}$-invariant subspace for the hybrid system $\Sigma$. The restrictions of $A$ and $J$ to $\mathcal{P}$ and the linear maps induced by $A$ and $J$ on the quotient space $\mathcal{X} / \mathcal{P}$ respectively are

$$
\begin{aligned}
& \left.A\right|_{\mathcal{P}}=A_{P},\left.\quad J\right|_{\mathcal{P}}=J_{P} \\
& \left.A\right|_{\mathcal{X} / \mathcal{P}}=A_{E},\left.\quad J\right|_{\mathcal{X} / \mathcal{P}}=J_{E}
\end{aligned}
$$


Proof. By taking (14), (15), and (22) into account, one can show that (19) and (20) hold with $V=P, L_{A}=A_{P}$, $M=0$, and $L_{J}=J_{P}$, which proves (23). Moreover, the block diagonal structure of $A$ and $J$ proves (24).

Lemma 1 Let $\mathcal{V} \subseteq \mathcal{X}$ be an $\mathscr{H}$-controlled invariant subspace for the hybrid extended system $\Sigma$ such that $\mathcal{V} \oplus \mathcal{P}=\mathcal{X}$, with a basis matrix $V$ of the form

$$
V=\left[\begin{array}{ll}
V_{P}^{\top} & I
\end{array}\right]^{\top}
$$

where the partition of $V$ is consistent with that in (14)-(16). Let $F: \mathcal{X} \rightarrow \mathcal{U}$, accordingly partitioned as

$$
F=\left[\begin{array}{ll}
F_{P} & F_{E}
\end{array}\right],
$$

be such that (21) holds. Then, the linear map $F^{\prime}: \mathcal{X} \rightarrow \mathcal{U}$, given by

$$
F^{\prime}=\left[\begin{array}{ll}
F_{P}+X & F_{E}-X V_{P}
\end{array}\right],
$$

where $V_{P}, F_{P}, F_{E}$ are as in (25), (26) and $X$ is any matrix of appropriate dimensions, is such that $\left(A+B F^{\prime}\right) \mathcal{V} \subseteq \mathcal{V}$.

Proof. First, note that (25) can be assumed as a basis matrix of $\mathcal{V}$ without loss of generality, since $\mathcal{V}$ is a direct summand of $\mathcal{P}$. Then, (21) is equivalent to the existence of a matrix $L_{F}$ such that

$$
\left[\begin{array}{cc}
A_{P}+B_{P} F_{P} & B_{P} F_{E} \\
0 & A_{E}
\end{array}\right]\left[\begin{array}{c}
V_{P} \\
I
\end{array}\right]=\left[\begin{array}{c}
V_{P} \\
I
\end{array}\right] L_{F},
$$

where (14), (25), and (26) have been taken into account. In particular, (28) holds with $L_{F}=A_{E}$ and, therefore, it is equivalent to

$$
\left(A_{P}+B_{P} F_{P}\right) V_{P}+B_{P} F_{E}=V_{P} A_{E} .
$$

A similar reasoning shows that $\left(A+B F^{\prime}\right) \mathcal{V} \subseteq \mathcal{V}$ holds if and only if there exists $L_{F}^{\prime}$ such that

$$
\begin{aligned}
& {\left[\begin{array}{cc}
A_{P}+B_{P} F_{P}+B_{P} X & B_{P} F_{E}-B_{P} X V_{P} \\
0 & A_{E}
\end{array}\right]\left[\begin{array}{c}
V_{P} \\
I
\end{array}\right]=} \\
& {\left[\begin{array}{c}
V_{P} \\
I
\end{array}\right] L_{F}^{\prime}}
\end{aligned}
$$

and (29) implies that (30) is met with $L_{F}^{\prime}=A_{E}$.

Theorem 2 Given the plant $\Sigma_{P}$ and the exogenous system $\Sigma_{E}$, consider the hybrid extended system $\Sigma$. Let $\left(A_{P}, B_{P}\right)$ be reachable and $(A, E)$ be observable. Problem 1 has a solution if there exists an $\mathscr{H}$-controlled invariant subspace $\mathcal{V} \subseteq \mathcal{X}$ for the hybrid extended system $\Sigma$, such that

$\mathcal{S} 1 . \mathcal{V} \oplus \mathcal{P}=\mathcal{X}$

$\mathcal{S} 2 . \mathcal{V} \subseteq \mathcal{E}$.

Proof. Let $\mathcal{V} \subseteq \mathcal{X}$ be an $\mathscr{H}$-controlled invariant subspace for the hybrid system $\Sigma$ satisfying Conditions $\mathcal{S} 1$ and $\mathcal{S} 2$. Let $F: \mathcal{X} \rightarrow \mathcal{U}$, partitioned as in (26), be such that $\mathcal{V}$ is $(A+B F)$-invariant and $J_{P} e^{\left(A_{P}+B_{P} F_{P}\right) \tau}$ is Schur stable. Note that the two requirements on $F$ are compatible by Lemma 1 and that stabilization of $J_{P} e^{\left(A_{P}+B_{P} F_{P}\right) \tau}$ is feasible by reachability of $\left(A_{P}, B_{P}\right)$ and Proposition 6 in the Appendix. Let $G: \mathcal{Y} \rightarrow \mathcal{X}$ be such that $J e^{(A+G E) \tau}$ is Schur stable - stabilization of $J e^{(A+G E) \tau}$ is feasible by observability of $(A, E)$ and Proposition 7 in the Appendix. Then, it will be shown that the hybrid regulator $\Sigma_{R}$, where
$A_{R}=A+B F+G E, \quad B_{R}=-G$,

$J_{R}=J$,

$C_{R}=F$,

solves Problem 1. To this aim, it will be shown that there exists a subspace $\hat{\mathcal{V}}$ which is invariant under the linear map $\hat{J} e^{\hat{A} \tau}$ and which satisfies Conditions $\mathcal{C} 1-\mathcal{C} 3$ of Theorem 1 , provided that the hybrid autonomous system $\hat{\Sigma}$ includes the specific regulator thus devised. By replacing (31)-(33) in (2)-(3), one gets

$$
\begin{aligned}
A_{L} & =\left[\begin{array}{cc}
A_{P} & B_{P} F \\
G C_{P} & A+B F+G E
\end{array}\right], \quad B_{L}=\left[\begin{array}{c}
0 \\
-G
\end{array}\right], \\
J_{L} & =\left[\begin{array}{cc}
J_{P} & 0 \\
0 & J
\end{array}\right] .
\end{aligned}
$$

Then, by replacing (34), (35), and (4) in (5)-(7), one gets

$$
\begin{aligned}
& \hat{A}=\left[\begin{array}{ccc}
A_{P} & B_{P} F & 0 \\
G C_{P} & A+B F+G E & -G C_{E} \\
0 & 0 & A_{E}
\end{array}\right], \\
& \hat{J}=\left[\begin{array}{ccc}
J_{P} & 0 & 0 \\
0 & J & 0 \\
0 & 0 & J_{E}
\end{array}\right], \\
& \hat{E}=\left[\begin{array}{lll}
-C_{P} & 0 & C_{E}
\end{array}\right] .
\end{aligned}
$$

As noted earlier, since $\mathcal{V}$ is a direct summand of $\mathcal{P}$, there is no loss of generality in assuming that a basis matrix $V$ of $\mathcal{V}$ has the structure shown in (25). Hence, it will be shown that a subspace $\hat{\mathcal{V}}$ satisfying the required conditions is

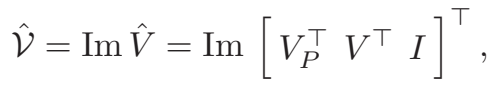

where the partition is consistent with that in (36)-(38). By applying the state space basis transformation

$$
\hat{T}=\left[\begin{array}{lll}
I & 0 & 0 \\
0 & 0 & I \\
0 & I & 0
\end{array}\right],
$$

partitioned accordingly, for the matrices of the hybrid autonomous system $\hat{\Sigma}$ in the new coordinates, one gets

$$
\begin{aligned}
& \hat{A}^{\prime}=\hat{T}^{-1} \hat{A} \hat{T}=\left[\begin{array}{ccc}
A_{P} & 0 & B_{P} F \\
0 & A_{E} & 0 \\
G C_{P} & -G C_{E} & A+B F+G E
\end{array}\right], \\
& \hat{J}^{\prime}=\hat{T}^{-1} \hat{J} \hat{T}=\left[\begin{array}{ccc}
J_{P} & 0 & 0 \\
0 & J_{E} & 0 \\
0 & 0 & J
\end{array}\right], \\
& \hat{E}^{\prime}=\hat{E} \hat{T}=\left[-C_{P} C_{E} 0\right]
\end{aligned}
$$

and, as far as the basis matrix of $\hat{\mathcal{V}}$, one gets

$$
\hat{V}^{\prime}=\hat{T}^{-1} \hat{V}=\left[\begin{array}{lll}
V_{P}^{\top} & I & V^{\top}
\end{array}\right]^{\top} .
$$

Hence, by taking (14)-(16) into account, (40)-(42) can be written in a more compact form as 


$$
\begin{aligned}
& \hat{A}^{\prime}=\left[\begin{array}{cc}
A & B F \\
-G E & A+B F+G E
\end{array}\right], \\
& \hat{J}^{\prime}=\left[\begin{array}{ll}
J & 0 \\
0 & J
\end{array}\right], \\
& \hat{E}^{\prime}=\left[\begin{array}{ll}
E & 0
\end{array}\right] .
\end{aligned}
$$

Consistently, the basis matrix $\hat{V}^{\prime}$ can be written as

$$
\hat{V}^{\prime}=\left[V^{\top} V^{\top}\right]^{\top}
$$

With reference to these coordinates, it is easy to show that $\hat{\mathcal{V}}$ is an $\mathscr{H}$-invariant subspace for $\hat{\Sigma}$. In fact: i) (12), where $\hat{A}$ and $\hat{W}$ are respectively replaced by $\hat{A}^{\prime}$, given by (44), and $\hat{V}^{\prime}$, given by (47), holds with $L_{\hat{A}}=A_{E}$, by virtue of $(A+B F)$-invariance of $\mathcal{V}$, in light of (14), (25), and (26); ii) (13), where $\hat{J}$ and $\hat{W}$ are respectively replaced by $\hat{J}^{\prime}$, given by (45), and $\hat{V}^{\prime}$, given by (47), holds with $L_{\hat{J}}=J_{E}$, by virtue of $J$-invariance of $\mathcal{V}$, in light of (15) and (25). Consequently, $\hat{\mathcal{V}}$ is invariant under the linear map $\hat{J} e^{\hat{A} \tau}$ by Proposition 2 . In order to show that Condition $\mathcal{C} 1$ of Theorem 1 is satisfied, note that, with respect to the original coordinates, the subspace $\hat{\mathcal{P}}$ is defined by (8). By applying the state space basis transformation $\hat{T}$ to the consistent, more detailed partition of $\hat{P}$, one gets

$$
\hat{P}^{\prime}=\hat{T}^{-1} \hat{P}=\hat{T}^{-1}\left[\begin{array}{lll}
I & 0 & 0 \\
0 & I & 0
\end{array}\right]^{\top}=\left[\begin{array}{lll}
I & 0 & 0 \\
0 & 0 & I
\end{array}\right]^{\top} .
$$

Taking (22) into account, $\hat{P}^{\prime}$ can be written in a more compact form as

$$
\hat{P}^{\prime}=\left[\begin{array}{ll}
P & 0 \\
0 & I
\end{array}\right] \text {. }
$$

The comparison between the basis matrices $\hat{V}^{\prime}$ and $\hat{P}^{\prime}$ respectively shown in (47) and (48) - in light of Condition $\mathcal{S} 1$, proves that Condition $\mathcal{C} 1$ of Theorem 1 is fulfilled. In order to show that Condition $\mathcal{C} 2$ of Theorem 1 is also met, first, note that $\hat{E} \hat{V}=E V=0$, by virtue of (38), (39), (16), (25), and Condition $\mathcal{S} 2$, which means that $\hat{\mathcal{V}} \subseteq \hat{\mathcal{E}}$. Hence, $\hat{\mathcal{V}}$, as an $\hat{A}$-invariant subspace contained in $\hat{\mathcal{E}}$ is also contained in $\hat{\mathcal{O}}$, which is the maximal $\hat{A}$-invariant subspace contained in $\hat{\mathcal{E}}$. Therefore, $\hat{\mathcal{V}}$, as a $\hat{J} e^{\hat{A} \tau}$-invariant subspace contained in $\hat{\mathcal{O}}$, is contained in $\hat{\mathcal{Q}}$, which is the maximal $\hat{J} e^{\hat{A} \tau}$-invariant subspace contained in $\hat{\mathcal{O}}$. In order to show that Condition $\mathcal{C} 3$ of Theorem 1 is satisfied, first note that $\hat{\mathcal{X}} / \hat{\mathcal{V}}=\hat{\mathcal{P}}$, since, as was shown above, $\hat{\mathcal{V}}$ and $\hat{\mathcal{P}}$ are direct summands. Hence, in light of Property 1, proving that Condition $\mathcal{C} 3$ holds reduces to proving that $J_{L} e^{A_{L} \tau}$ is Schur stable. To this aim, a more detailed partition of (34) and (35) is considered, where $F$ is partitioned as in (26) and $G$ is consistently partitioned as

Thus,

$$
G=\left[G_{P}^{\top} G_{E}^{\top}\right]^{\top}
$$

$$
\begin{aligned}
& A_{L}= \\
& {\left[\begin{array}{ccc}
A_{P} & B_{P} F_{P} & B_{P} F_{E} \\
G_{P} C_{P} & A_{P}+B_{P} F_{P}-G_{P} C_{P} & B_{P} F_{E}+G_{P} C_{E} \\
G_{E} C_{P} & -G_{E} C_{P} & A_{E}+G_{E} C_{E}
\end{array}\right],}
\end{aligned}
$$

$J_{L}=\left[\begin{array}{ccc}J_{P} & 0 & 0 \\ 0 & J_{P} & 0 \\ 0 & 0 & J_{E}\end{array}\right]$,

where (14)-(16), (26), and (49) have been considered. Then, by performing the state space basis transformation

$$
S_{L}=\left[\begin{array}{lll}
I & 0 & 0 \\
I & I & 0 \\
0 & 0 & I
\end{array}\right]
$$

one gets

$$
\begin{aligned}
& A_{L}^{\prime}=S_{L}^{-1} A_{L} S_{L}= \\
& {\left[\begin{array}{ccc}
A_{P}+B_{P} F_{P} & B_{P} F_{P} & B_{P} F_{E} \\
0 & A_{P}-G_{P} C_{P} & G_{P} C_{E} \\
0 & -G_{E} C_{P} & A_{E}+G_{E} C_{E}
\end{array}\right],}
\end{aligned}
$$$$
J_{L}^{\prime}=S_{L}^{-1} J_{L} S_{L}=J_{L}
$$

Again, in light of (14)-(16), (26), and (49), (50) and (51) can be written in a more compact form as

$$
\begin{aligned}
A_{L}^{\prime} & =\left[\begin{array}{cc}
A_{P}+B_{P} F_{P} & B_{P} F \\
0 & A+G E
\end{array}\right], \\
J_{L}^{\prime} & =\left[\begin{array}{cc}
J_{P} & 0 \\
0 & J
\end{array}\right] .
\end{aligned}
$$

Therefore, from (52) and (53), it ensues that

$$
J_{L}^{\prime} e^{A_{L}^{\prime} \tau}=\left[\begin{array}{cc}
J_{P} e^{\left(A_{P}+B_{P} F_{P}\right) \tau} & * \\
0 & J e^{(A+G E) \tau}
\end{array}\right],
$$

where $*$ stands for a matrix block of no interest to this discussion. Finally, the upper block-triangular structure of $J_{L}^{\prime} e^{A_{L}^{\prime} \tau}$ and Schur stability of the matrix blocks on the main diagonal imply Schur stability of $J_{L} e^{A_{L} \tau}$.

Remark 2 The hybrid regulator $\Sigma_{R}$ devised in the proof of Theorem 2 satisfies a more general version of the internal model principle, compared to that originally given in (Francis et al., 1974), naturally adapted to hybrid systems with a continuous-time linear dynamics subject to periodic state jumps. In fact, the continuous-time dynamics of $\Sigma_{R}$ - represented by $A_{R}$ in (31) - includes the eigenstructure of the continuous-time dynamics of the exogenous system. This is shown by the fact that the subspace $\mathcal{V}$, as an $(A+B F)$-invariant subspace contained in $\mathcal{E}$, is also an $(A+B F+G E)$-invariant subspace and, for the same reason, $\left.(A+B F+G E)\right|_{\mathcal{V}}=\left.(A+B F)\right|_{\mathcal{V}}$, where the latter has been proven to coincide with $A_{E}-i . e$. , the continuoustime dynamic matrix of the exogenous system. Moreover, the jump dynamics of $\Sigma_{R}$ - represented by $J_{R}$ in (32) - includes that of the exogenous system - namely, $J_{E}$.

Remark 3 The sufficient condition expressed by Theorem 2 is equivalent to the existence of matrices $V_{P}$ and $M$, of appropriate dimensions, which solve the set of linear algebraic matrix equations

$\begin{aligned} A_{P} V_{P} & =V_{P} A_{E}+B_{P} M, \\ J_{P} V_{P} & =V_{P} J_{E} \\ C_{P} V_{P} & =C_{E}\end{aligned}$ 
with $A_{P}, B_{P}, J_{P}, C_{P}, A_{E}, J_{E}$, and $C_{E}$ given. In fact, the condition that $\mathcal{V}$ is a direct summand of $\mathcal{P}$ allows the structure of a basis matrix of $\mathcal{V}$ to be assumed as in (25). Hence, (54), (55) express the condition of $\mathscr{H}$-controlled invariance in a coordinate-dependent setting. Further, (56) expresses the condition that $\mathcal{V}$ is contained in $\mathcal{E}$. Note that (55) generically has the unique solution $V_{P}=0$, but $V_{P}=0$ does not solve (56) except in the trivial case where $C_{E}=0$. However, if $J_{P}$ and $J_{E}$ have some common eigenvalue, (55) has infinitely many solutions and some of them may be solutions of (56) and, for some matrix $M$, of (54) too.

By using the vectorization operator, denoted by $\operatorname{vec}(\cdot)$, and the Kronecker product, denoted by $\otimes,(54)-(56)$ can be written as the system of $n_{E} \times\left(2 n_{P}+q\right)$ linear algebraic scalar equations in $n_{E} \times n_{P}$ unknowns expressed, in compact form, as

$$
\begin{gathered}
{\left[\begin{array}{c}
\left(I_{n_{E}} \otimes A_{P}\right)-\left(A_{E}^{\top} \otimes I_{n_{P}}\right) \\
\left(I_{n_{E}} \otimes J_{P}\right)-\left(J_{E}^{\top} \otimes I_{n_{P}}\right) \\
\left(I_{n_{E}} \otimes C_{P}\right)
\end{array}\right]} \\
\operatorname{vec}\left(V_{P}\right)= \\
{\left[\begin{array}{c}
\left(I_{n_{E}} \otimes B_{P}\right) \operatorname{vec}(M) \\
0 \\
\operatorname{vec}\left(C_{E}\right)
\end{array}\right],}
\end{gathered}
$$

where $\operatorname{vec}(M)$ is a vector of $n_{E} \times p$ free parameters. Any solution of the system of linear equations (57), for some choice of the free parameters, gives the matrix $V_{P}$ to be used to construct - according to (25) - the basis matrix $V$ of an $\mathscr{H}$-controlled invariant subspace $\mathcal{V}$ contained in $\mathcal{E}$.

Remark 4 The major interest of the solvability condition expressed by Theorem 2 is connected to the synthesis of the hybrid feedback regulator. In the first place, provided that a subspace $\mathcal{V}$ satisfying the requirements of Theorem 2 exist and be known, the proof exactly and straightforwardly shows how to construct the hybrid regulator. Secondly, the time period between two consecutive jumps is not involved in the solvability condition expressed by Theorem 2. This means that, if such solvability condition is satisfied, then Problem 1 is solvable for any time period $\tau$ (of finite length). Nonetheless, the sequence of jump times is supposed to be available to the hybrid feedback regulator consequently designed.

A more extensive (as will be clear from the following) and yet only sufficient condition to solve Problem 1 is established by the next theorem.

Theorem 3 Given the plant $\Sigma_{P}$ and the exogenous system $\Sigma_{E}$, consider the hybrid extended system $\Sigma$. Let $(A, E)$ be observable. Problem 1 has a solution if there exists a $J e^{(A+B F) \tau}$-invariant subspace $\mathcal{V} \subseteq \mathcal{X}$ such that Conditions $\mathcal{S} 1$ and $\mathcal{S} 2$ hold $-F$ being a linear map, partitioned as in (26), which makes $J_{P} e^{\left(A_{P}+B_{P} F_{P}\right) \tau}$ Schur stable.

Proof. The proof follows the same lines as that of Theorem 2. In particular, the hybrid feedback regulator $\Sigma_{R}$ is defined according to (31)-(33), with the only difference that the linear map $F$ is picked as specified in the statement of Theorem 3.

Remark 5 The condition of Theorem 3 is less restrictive than that of Theorem 2, since any subspace $\mathcal{V}$ satisfying the requisites of Theorem 2 also satisfies the requisites of Theorem 3, while the converse is not true in general. Actually, if $\mathcal{V}$ is an $\mathscr{H}$-controlled invariant subspace of the hybrid extended system $\Sigma$, on the assumptions of Theorem 2, there exists a linear map $F$ such that $(A+B F) \mathcal{V} \subseteq \mathcal{V}$ and $J_{P} e^{\left(A_{P}+B_{P} F_{P}\right) \tau}$ is Schur stable. Hence, the subspace $\mathcal{V}$, which is simultaneously $(A+B F)$-invariant and $J$ invariant, can also be shown to be invariant under the linear map $J e^{(A+B F) \tau}$, for any given positive real constant $\tau$, by applying the same arguments considered in the proof of Proposition 2.

In light of the previous remarks, the comparison between Theorem 2 and Theorem 3 shows that the counterpart of relaxing the condition of $\mathscr{H}$-controlled invariance to a condition of invariance with respect to the state transition matrix over one period is that of including the to-be-designed linear map $F$ in the condition itself, which is a circumstance similar (although less manifest) to that presented in Theorem 1, where all the to-be-designed linear maps defining the regulator are included in the necessary and sufficient condition.

\section{A Constructive Condition for the Resolving Subspace}

This section introduces a necessary and sufficient condition to compute the $\mathscr{H}$-controlled invariant subspace $\mathcal{V}$ that plays a key role in Theorem 2. To this aim, the maximal $\mathscr{H}$ controlled invariant subspace contained in the null space of the output of the hybrid system $\Sigma$ is defined through the following reasoning.

Proposition 5 The set of all $\mathscr{H}$-controlled invariant subspaces contained in the null space of the output of the hybrid system $\Sigma$ is an upper semilattice with respect to the sum and the inclusion of subspaces - such semilattice is denoted by $\Phi_{\mathscr{H}}(\mathcal{E})$.

Proof. First, note that $\mathscr{H}$-controlled invariance involves simultaneous $(A, \mathcal{B})$-controlled invariance and $J$-invariance. Concerning $(A, \mathcal{B})$-controlled invariance, standard arguments of linear algebra show that the set of all $(A, \mathcal{B})$ controlled invariant subspaces contained in a subspace $\mathcal{E}$ is an upper semilattice with the sum as binary operation and the inclusion as partial ordering relation. As to $J$-invariance, it can likewise be shown that the set of all $J$-invariant subspaces contained in $\mathcal{E}$ is an upper semilattice with respect to the sum and the inclusion. Consequently, the set of all subspaces contained in $\mathcal{E}$, with the property of being both $(A, \mathcal{B})$-controlled invariant and $J$-invariant, is an upper semilattice with respect to the sum and the inclusion.

Definition 3 The supremum of $\Phi_{\mathscr{H}}(\mathcal{E})$ is called the maximal $\mathscr{H}$-controlled invariant subspace contained in the kernel of the output of the hybrid system $\Sigma$ and is denoted by $\mathcal{V}^{*}$.

The subspace $\mathcal{V}^{*}$, introduced in Definition 3, can be computed through the algorithm presented below. As to the formalization of the algorithm, $\max \mathcal{J}(J, \mathcal{K})$, where $\mathcal{K}$ denotes a subspace of $\mathcal{X}$, stands for the maximal $J$-invariant subspace contained in $\mathcal{K}$. Moreover, $\max \mathcal{V}(A, \mathcal{B}, \mathcal{W})$, where $\mathcal{B}$ and $\mathcal{W}$ denote subspaces of $\mathcal{X}$, stands for the maximal $(A, \mathcal{B})$-controlled invariant subspace contained in $\mathcal{W}$. The 
subspaces $\max \mathcal{J}(J, \mathcal{K})$ and $\max \mathcal{V}(A, \mathcal{B}, \mathcal{W})$ can be respectively computed as in (Basile and Marro, 1992, Algorithms 3.2-2 and 4.1-2).

Algorithm 1 The maximal $\mathscr{H}$-controlled invariant subspace contained in the kernel of the output of the hybrid system $\Sigma$ - namely, $\mathcal{V}^{*}$ - is the last term of the sequence $\mathcal{J}_{0}=\max \mathcal{J}(J, \mathcal{E}), \mathcal{V}_{i}=\max \mathcal{V}\left(A, \mathcal{B}, \mathcal{J}_{i-1}\right)$, $\mathcal{J}_{i}=\max \mathcal{J}\left(J, \mathcal{V}_{i}\right), i=1, \ldots, k$, where $k \leq n-1$ is the least integer such that $\mathcal{J}_{k}=\mathcal{V}_{k}$.

The following statements will show how the subspace $\mathcal{V}^{*}$, which has been introduced by Definition 3 and which can be computed by means of Algorithm 1, can be used, under certain conditions, to compute an $\mathscr{H}$-controlled invariant subspace $\mathcal{V}$, satisfying the conditions of Theorem 2 .

Lemma 2 Consider the hybrid extended system $\Sigma$, the subspace $\mathcal{P}$, and the subspace $\mathcal{V}^{*}$. Let

$$
\mathcal{V}^{*}+\mathcal{P}=\mathcal{X}
$$

hold. Let the linear map $F: \mathcal{X} \rightarrow \mathcal{U}$ be such that $(A+B F) \mathcal{V}^{*} \subseteq \mathcal{V}^{*}$. Perform the state space basis transformation $Q=\left[\begin{array}{lll}Q_{1} & Q_{2} & Q_{3}\end{array}\right]$, where $\operatorname{Im} Q_{1}=\mathcal{V}^{*} \cap \mathcal{P}$, $\operatorname{Im}\left[Q_{1} Q_{2}\right]=\mathcal{V}^{*}$, and $\operatorname{Im}\left[Q_{1} Q_{3}\right]=\mathcal{P}$. Then, with respect to the new coordinates,

$$
\begin{aligned}
& A^{\prime}+B^{\prime} F^{\prime}=Q^{-1}(A+B F) Q= \\
& {\left[\begin{array}{ccc}
A_{11}^{\prime}+B_{1}^{\prime} F_{1}^{\prime} & A_{12}^{\prime}+B_{1}^{\prime} F_{2}^{\prime} & A_{13}^{\prime}+B_{1}^{\prime} F_{3}^{\prime} \\
0 & A_{22}^{\prime} & 0 \\
0 & 0 & A_{33}^{\prime}+B_{3}^{\prime} F_{3}^{\prime}
\end{array}\right],} \\
& J^{\prime}=Q^{-1} J Q=\left[\begin{array}{ccc}
J_{11}^{\prime} & J_{12}^{\prime} & J_{13}^{\prime} \\
0 & J_{22}^{\prime} & 0 \\
0 & 0 & J_{33}^{\prime}
\end{array}\right] .
\end{aligned}
$$

Proof. $\mathscr{H}$-invariance of $\mathcal{P}$ and the inclusion $\mathcal{B} \subseteq \mathcal{P}$ account for the zero matrices in the second block of rows of $A^{\prime}+B^{\prime} F^{\prime}$ and $J^{\prime}$, respectively. $\mathscr{H}$-controlled invariance of $\mathcal{V}^{*}$ accounts for the zero matrices in the last block of rows of $J^{\prime}$, and, in particular, $(A+B F)$-invariance of $\mathcal{V}^{*}$ accounts for the zero matrices in the last block of rows of $A^{\prime}+B^{\prime} F^{\prime}$.

Theorem 4 Given the plant $\Sigma_{P}$ and the exogenous system $\Sigma_{E}$, consider the hybrid extended system $\Sigma$, the subspace $\mathcal{P}$, and the subspace $\mathcal{V}^{*}$. There exists an $\mathscr{H}$-controlled invariant subspace of $\Sigma$ satisfying Conditions $\mathcal{S} 1$ and $\mathcal{S} 2$ of Theorem 2 if and only if (58) holds and there exixts a matrix $X$ that solves the linear matrix equations

$$
\begin{aligned}
& \left(A_{11}^{\prime}+B_{1}^{\prime} F_{1}^{\prime}\right) X-X A_{22}^{\prime}=-A_{12}^{\prime}-B_{1}^{\prime} F_{2}^{\prime}, \\
& J_{11}^{\prime} X-X J_{22}^{\prime}=-J_{12}^{\prime},
\end{aligned}
$$

where the $A_{11}^{\prime}, A_{22}^{\prime}, A_{12}^{\prime}, J_{11}^{\prime}, J_{22}^{\prime}, J_{12}^{\prime}, B_{1}^{\prime}, F_{1}^{\prime}$, and $F_{2}^{\prime}$ were introduced in Lemma 2.

Proof. If. Let (58) hold and let $X$ satisfy (61) and (62). It will be shown that the subspace

$$
\mathcal{V}=\operatorname{Im} V^{\prime}=\operatorname{Im}\left[\begin{array}{lll}
X^{\top} & I & 0
\end{array}\right]^{\top},
$$

where the partition considered in (63) is consistent with that shown in (59) and (60), is an $\mathscr{H}$-controlled invariant subspace of $\Sigma$ satisfying Conditions $\mathcal{S} 1$ and $\mathcal{S} 2$ of Theorem 2 .
To this purpose, first, observe that $\left(A^{\prime}+B^{\prime} F^{\prime}\right) V^{\prime}=V^{\prime} L_{F}$ holds with $L_{F}=A_{22}^{\prime}$ by virtue of (61), which implies that $\mathcal{V}$ is an $(A+B F)$-invariant subspace or, equivalently, an $(A, \mathcal{B})$-controlled invariant subspace. Secondly, note that $J^{\prime} V^{\prime}=V^{\prime} L_{J}$ holds with $L_{J}=J_{22}^{\prime}$ by virtue of (62), which implies that $\mathcal{V}$ is a $J$-invariant subspace. Thus, $\mathcal{V}$, as an $(A, \mathcal{B})$-controlled invariant subspace and a $J$-invariant subspace, is an $\mathscr{H}$-controlled invariant subspace of $\Sigma$. In addition, the comparison between the basis matrices of $\mathcal{V}$ and $\mathcal{P}$ with respect to the coordinates introduced in Lemma 2 shows that Condition $\mathcal{S} 1$ of Theorem 2 is met. Furthermore, the comparison between the basis matrices of $\mathcal{V}$ and $\mathcal{V}^{*}$ shows that $\mathcal{V} \subseteq \mathcal{V}^{*}$ and, consequently, also Condition $\mathcal{S} 2$ of Theorem 2 is fulfilled.

Only if. Let $\mathcal{V}$ be an $\mathscr{H}$-controlled invariant subspace of $\Sigma$ satisfying Conditions $\mathcal{S} 1$ and $\mathcal{S} 2$ of Theorem 2 . Hence, $\mathcal{V}^{*}$, which contains $\mathcal{V}$, satisfies (58). Then, consider the similarity transformation $Q$ introduced in Lemma 2 and choose $Q_{2}$ as a basis matrix of $\mathcal{V}$. Therefore, $A_{12}^{\prime}-B_{1}^{\prime} F_{2}^{\prime}=0$ in (59), which implies that (61) is satisfied with $X=0$. For the same reason, $J_{12}^{\prime}=0$ in (60), which implies that $X=0$ also satisfies (62).

It is worth noting that Theorem 4 and, more generally, all the arguments developed in this section only deal with structural notions, thus leaving stability apart from consideration. In fact, the only requirement on the linear map $F$ considered in Lemma 2 and Theorem 4 is to render $\mathcal{V}^{*}$ an $(A+B F)$-invariant subspace - no stabilization properties are required. Therefore, once the subspace $\mathcal{V}$ has been determined, if possible, according to Theorem $4, \mathcal{V}$ can be used to synthesize the feedback regulator according to Theorem 2, which means, in particular, that a different linear map $F$ can be determined to this aim. Namely, a different linear map $F$, partitioned as in (26), can be used to render $J_{P} e^{\left(A_{P}+B_{P} F_{P}\right) \tau}$ Schur stable and to render $\mathcal{V}$ an $(A+B F)$-invariant subspace.

\section{A Numerical Example}

The aim of this section is to illustrate the devised synthesis procedure through a worked out numerical example. Numerical computations are made with Matlab and Matlab-based software for the geometric approach available with (Basile and Marro, 1992), while symbolic computations are done with Maple. Let Problem 1 be stated for the plant $\Sigma_{P}$, where

$$
\begin{aligned}
& A_{P}=\left[\begin{array}{ccc}
-4 & 1 & 0 \\
5 & -3 & 1 \\
1 & 0 & -4
\end{array}\right], B_{P}=\left[\begin{array}{cc}
1 & -1 \\
0 & 1 \\
0 & 0
\end{array}\right], J_{P}=\left[\begin{array}{lll}
5 & 0 & 0 \\
0 & 5 & 0 \\
0 & 0 & 5
\end{array}\right], \\
& C_{P}=\left[\begin{array}{ccc}
-1 & -3 & 2 \\
0 & -3 & 0
\end{array}\right],
\end{aligned}
$$

and the exogenous system $\Sigma_{E}$, where

$$
A_{E}=\left[\begin{array}{ll}
0 & 1 \\
0 & 0
\end{array}\right], J_{E}=\left[\begin{array}{ll}
5 & 0 \\
0 & 5
\end{array}\right], C_{E}=\left[\begin{array}{cc}
-2 & 0 \\
0 & 3
\end{array}\right] \text {. }
$$

Let $\tau=1$. Note that the spectrum of $J_{E} e^{A_{E} \tau}$ is $\{5,5\}$ and, as required, lies outside the open unit disc of $\mathbb{C}$. 


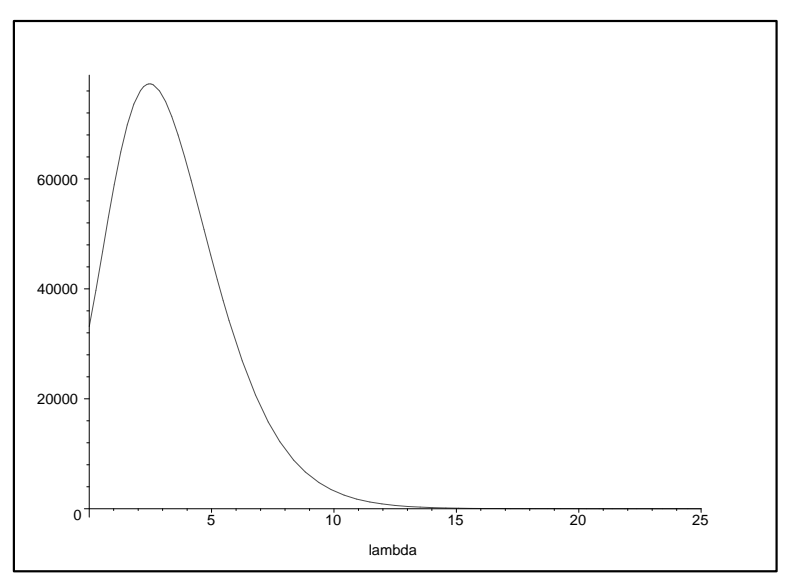

Fig. 1. Function $f(\lambda)-$ Plot

In order to check on whether the sufficient conditions for the output regulation problem to be solvable are satisfied, the hybrid extended system $\Sigma$ is constructed. With reference to the sufficient conditions stated in Theorem 4, the subspace $\mathcal{V}^{*}$ is computed through Algorithm 1, while the subspace $\mathcal{P}$ is defined according to (22). Hence,

$$
\mathcal{V}^{*}=\operatorname{Im}\left[\begin{array}{ccccc}
2 & 0 & 1 & 0 & 0 \\
4 & 0 & 1 & 1 & 0 \\
5 & -1 & 1 & 0 & 1
\end{array}\right]^{\top}
$$

and $\mathcal{P}=\operatorname{Im}\left[I_{3} 0_{3 \times 2}\right]^{\top}$. As is easy to check, $\mathcal{V}^{*}+\mathcal{P}=\mathcal{X}$ and $\mathcal{V}^{*} \cap \mathcal{P} \neq\{0\}$. Moreover, (61)-(62) have a solution and it can be shown that an $\mathscr{H}$-controlled invariant subspace $\mathcal{V}$, contained in $\mathcal{E}$ and such that $\mathcal{V} \oplus \mathcal{P}=\mathcal{X}$ is

$$
\mathcal{V}=\operatorname{Im}\left[\begin{array}{ccccc}
4 & 0 & 1 & 1 & 0 \\
5 & -1 & 1 & 0 & 1
\end{array}\right]^{\top} \text {. }
$$

Hence, $\mathcal{V}$ is a subspace satisfying Conditions $\mathcal{S} 1$ and $\mathcal{S} 2$ of Theorem 2. Therefore, going ahead with the design of the hybrid regulator $\Sigma_{R}$ as specified in the proof of Theorem 2, a linear map $F$, partitioned as in (26), has to be found, so that $J_{P} e^{\left(A_{P}+B_{P} F_{P}\right) \tau}$, with $\tau=1$, is Schur stable and (21) holds. Note that $J_{P} e^{A_{P}}$ is not Schur stable, since its spectrum is $\{1.6090,0.0175,0.0742\}$. However, as is easy to check, the pair $\left(A_{P}, B_{P}\right)$ is reachable. Therefore, $F_{P}$ can be obtained by applying the procedure shown in the proof of Proposition 6 in the Appendix. By exploiting cyclicity of $\left(A_{P}, B_{P}\right)$ with respect to the first column, say $b_{P}$, of $B_{P}$ and letting

$$
F_{P}=\left[\begin{array}{ccc}
f_{1} & f_{2} & f_{3} \\
0 & 0 & 0
\end{array}\right]
$$

be the feedback that assigns the spectrum of (distinct) eigenvalues $\left\{\lambda_{1}, \lambda_{2}, \lambda_{3}\right\}$ to $\left(A_{P}+B_{P} F_{P}\right)$, it follows that $\left\|J_{P} e^{\left(A_{P}+B_{P} F_{P}\right) \tau}\right\|=\left\|J_{P} S^{-1} Q^{-1} e^{\Lambda \tau} Q S\right\|$, where $\Lambda=\operatorname{diag}\left\{\lambda_{1}, \lambda_{2}, \lambda_{3}\right\}, S$ is such that the pair $\left(S^{-1} A_{P} S, S^{-1} b_{P}\right)$ is in controllable canonical form and

$$
Q=\left[\begin{array}{ccc}
1 & 1 & 1 \\
\lambda_{1} & \lambda_{2} & \lambda_{3} \\
\lambda_{1}^{2} & \lambda_{2}^{2} & \lambda_{3}^{2}
\end{array}\right]
$$

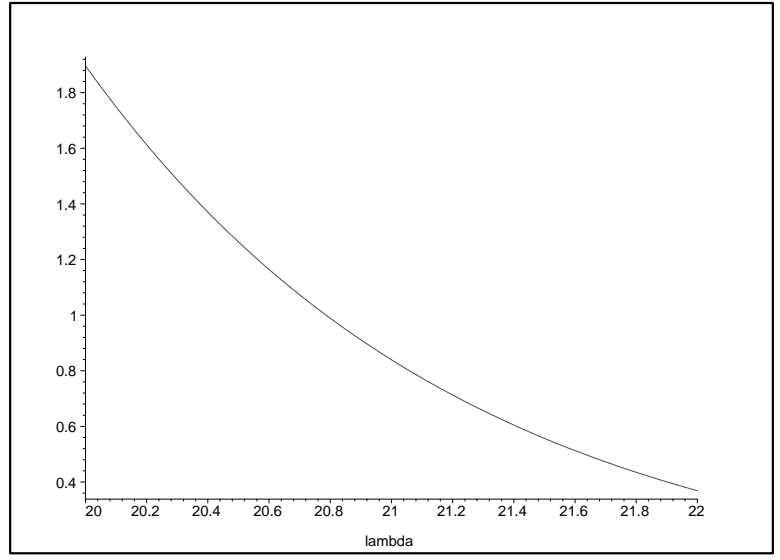

Fig. 2. Function $f(\lambda)-$ Zoomed plot

Letting $\lambda_{i}=-\lambda-i+1$ for $i=1,2,3$, it ensues that, with $\tau=1$,

$$
\begin{aligned}
& \left\|J_{P} e^{\left(A_{P}+B_{P} F_{P}\right)}\right\| \leq \\
& \left\|J_{P}\right\|\left\|S^{-1}\right\|\|S\|\left\|Q^{-1}\right\| \mathscr{F}\|Q\|_{\mathscr{F}} e^{-\lambda}=f(\lambda) .
\end{aligned}
$$

By using Maple to get a symbolic expression of $Q^{-1}$ and of the Frobenius norms of $Q$ and $Q^{-1}$, it is possible to derive an explicit expression of the function $f(\lambda)$ and to plot it for $\lambda \in \mathbb{R}$ (see Fig. 1 and Fig. 2). Note that the motivation for the use of the Frobenius norm is to limit the complexity of such expression. In particular, the inspection of the zoomed plot (Fig. 2), shows that, for instance,

$$
\left\|J_{P} e^{\left(A_{P}+B_{P} F_{P}\right)}\right\| \leq f(21) \leq 1 .
$$

Clearly, the choice $\lambda=21$ is conservative, due to the way the upper bound $f(\lambda)$ for $\left\|J_{P} e^{\left(A_{P}+B_{P} F_{P}\right)}\right\|$ has been constructed and, as a matter of fact, the order of magnitude of the eigenvalues of $J_{P} e^{A_{P}+B_{P} F_{P}}$, with that choice, is $10^{-7}$. However, in order to reduce conservativeness, one can use, e.g., an iterative dichotomic method on the interval $[0,21]$ to modify $\lambda$, evaluating at each step the eigenvalues of $J_{P} e^{\left(A_{P}+B_{P} F_{P}\right)}$ to check Schur stability. In this way, $\lambda=2$ is found to be a possible choice, while $\lambda=1$ is not. The resulting feedback $F_{P}$ is obtained by letting $f_{1}=2$, $f_{2}=-1, f_{3}=0$. Thus, the spectrum of $J_{P} e^{\left(A_{P}+B_{P} F_{P}\right)}$ is $\{0.6767,0.2489,0.0916\}$. Then, by imposing (21), one gets

$$
F=\left[\begin{array}{ccccc}
2 & -1 & 0 & -13 & -15 \\
0 & 0 & 0 & -21 & -29
\end{array}\right]
$$

with simple computations. Then, a linear map $G$ has to be found, such that $J e^{(A+G E) \tau}$, with $\tau=1$, is Schur stable. Again, it is easy to check that $(A, E)$ is observable, which means that Proposition 7 applies. By searching for a map $G$ that assigns the spectrum $\{-\lambda,-\lambda-1, \ldots,-\lambda-4\}$ to $A+G E$ and by applying the same techniques as before, it is possible to find a function $g(\lambda)$ that gives a (conservative) upper bound for $\left\|J e^{(A+G E) \tau}\right\|$, with $\tau=1$. The inspection of the plot of $g(\lambda)$ for $\lambda \in \mathbb{R}$ shows that, e.g., $\left\|J e^{(A+G E)}\right\| \leq g(36) \leq 1$ (this plot is qualitatively similar to that of $f(\lambda)$ and has not been reported here for the sake of space). By applying the dichotomic procedure mentioned above, $\lambda=3$ is obtained. Then, the resulting map $G$ is 


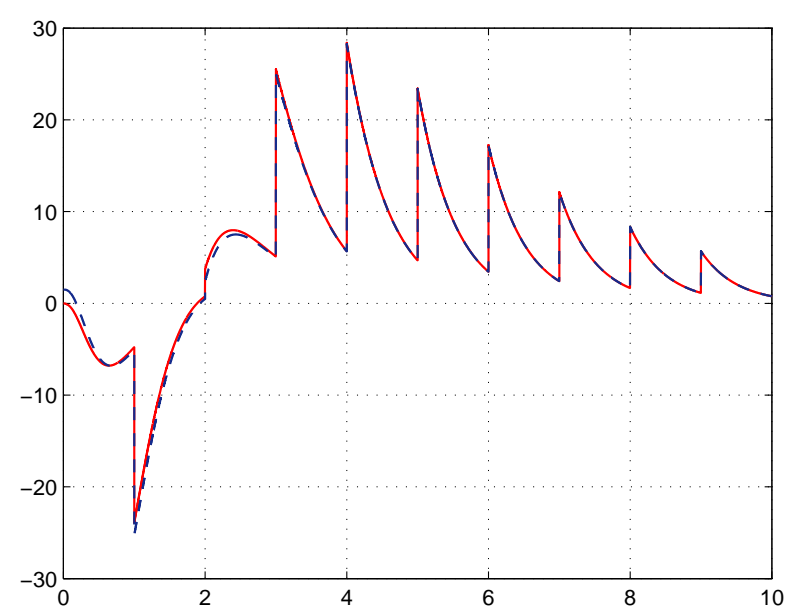

Fig. 3. Output regulation error - amplitude vs. time (s)

$$
G=\frac{1}{891}\left[\begin{array}{ccccc}
-3305 & -9467 & -1157 & -8459 & 41580 \\
0 & 0 & 0 & 0 & 0
\end{array}\right]^{\top},
$$

and the spectrum of $J e^{(A+G E) \tau}$ is $\{0.2489,0.0916,0.0046,0.0124,0.0337\}$. Thus, all the elements for determining the hybrid regulator $\Sigma_{R}$, according to (31)-(33), have been computed.

Finally, it is shown how the hybrid regulator thus devised actually achieves the design objectives. As to global asymptotic stability of the closed-loop dynamics (Requirement $\mathcal{R} 1$ of Problem 1), by computing $A_{L}$ and $J_{L}$ according to (2) and (3), one can see that the spectrum of $J_{L} e^{A_{L} \tau}$ is the union (with repetition count) of those of $J_{P} e^{\left(A_{P}+B_{P} F_{P}\right) \tau}$ and $J e^{(A+G E) \tau}$, which implies that $J_{L} e^{A_{L} \tau}$ is Schur stable. As to asymptotic tracking of the reference (Requirement $\mathcal{R} 2$ of Problem 1), the performance of the feedback hybrid system thus designed can be illustrated, for instance, by running the simulation described below. The simulation time is assumed to be $10 \mathrm{~s}$. The initial state of the hybrid autonomous system $\hat{\Sigma}$, defined as in (5)-(7), is assumed to

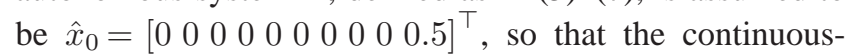
time dynamics of the exogenous system generates a ramp at the first output component and a step at the second output component. Moreover, these reference signals are affected by discontinuities as a consequence of the fact that the components of the state are multiplied by 5 every $1 \mathrm{~s}$. The behavior of the two components of the regulation error, converging to zero as the time increases, is shown in Fig. 3: the first and the second component are represented by the solid and the dashed line, respectively.

To conclude this section, it is worth analyzing some numerical examples available from the literature - in particular, the example worked out in (Marconi and Teel, 2010), also revisited in (Carnevale et al., 2012a), and the one described in (Carnevale et al., 2016) - in light of the results presented in this work. As to the numerical example of (Marconi and Teel, 2010; Carnevale et al., 2012a), this does not lend itself to be solved with the techniques devised in this work for the following reason. As mentioned in the Introduction, the problem formulation considered in the abovementioned papers concerns not only asymptotic tracking of the reference generated by the exogenous system, but also decoupling of another signal, still generated by the exogenous system but directly affecting the plant. Actually, the numerical example at issue refers to a special case of that problem statement, which reduces to the sole decoupling of the signal acting on the plant, while the to-be-tracked reference is zero. Consequently, the assumptions under which such decoupling problem makes sense are different from those under which the problem dealt with in this work, which just concerns asymptotic tracking, is meaningful. In particular, the assumption that the pair $(A, E)$ is observable is not true for the considered numerical example. Consequently, the arbitrary assignment of the spectrum of $A+G E$ required in the constructive proof of Theorem 2 (see also Proposition 7 in the Appendix) is prevented. As far as the numerical example worked out in (Carnevale et al., 2016) is concerned, this is indeed formulated as a typical asymptotic reference tracking problem. However, there is a structural obstruction to achieving a solution through the method devised in this work. As it turns out, the dimension of the subspace $\mathcal{V}^{*}$ is equal to 1 , which implies that the subspace $\mathcal{V}$, contained in $\mathcal{V}^{*}$, cannot be a direct summand of $\mathcal{P}$, whose dimension is 3 , in the state space $\mathcal{X}$ of dimension 5 . This means that Condition $\mathcal{S} 1$ of Theorem 2 is not satisfied. Nevertheless, it is worth remarking that the structure of the regulators considered in the papers cited above (not only the methodology devised to design them) is noticeably more complex than the one postulated in this work.

\section{Conclusions}

In this work, the problem of achieving output regulation with closed-loop global asymptotic stability in hybrid systems with a continuous-time linear dynamics subject to periodic state jumps, by means of a feedback regulator with the same characteristics, has been investigated. Starting from a necessary and sufficient condition where the matrices of the to-be-designed regulator are implicitly considered, the discussion develops so as to lead to sufficient conditions on which a complete and viable synthesis procedure is based. A numerical example has been worked out in order to illustrate how to apply the devised method.

\section{A Appendix. Stabilization of Hybrid Systems with Pe- riodic State Jumps}

Reachability of the flow dynamics of the hybrid plant and observability of the flow dynamics of the hybrid extended system are technical assumptions that, in Theorem 2, guarantee asymptotic stabilizability by means, respectively, of a timeinvariant state feedback or a time-invariant output injection which only act on the flow dynamics at issue. This makes it possible to synthesize the feedback regulator, whose defining matrices, in the formulation of Problem 1, are required to be time-invariant. Although different assumptions, milder than reachability and observability of the flow dynamics, are known to assure stabilizability of the hybrid system (see, e.g., Medina and Lawrence, 2009; Carnevale et al., 2012b), they cannot be used in herein, since either they lead to the synthesis of a time-varying stabilizing feedback or they require that the stabilizing feedback also acts on the jump dynamics. 
Indeed, the fact that reachability of the flow dynamics implies stabilizability by a time-invariant feedback that acts only on the flow dynamics has been shown in Carnevale et al. (2014b). In Proposition 6, a different proof of the same result is given by using inequalities between matrix norms and eigenvalue assignment (instead of linear matrix inequalities as in the above quoted paper). The main motivation for providing a new proof is the fact that it defines a quite practical synthesis procedure, which makes it possible to assign the eigenvalues of the compensated flow dynamics while stabilizing the hybrid system. Likewise, observability of the flow dynamics ensures that global asymptotic stability of the hybrid dynamics can be achieved via output injection, as can be shown by dual arguments. The computational procedure outlined in the main part of this paper is therefore completed. To avoid notation clutter, these results will be stated with direct reference to the hybrid systems whose stabilization ensures global asymptotic stability of the closed-loop hybrid dynamics considered in the output regulation problem.

Proposition 6 Consider the hybrid system $\Sigma_{P}$. Let $\left(A_{P}, B_{P}\right)$ be reachable. Then, there exists a linear map $F_{P}: \mathcal{X}_{p} \rightarrow \mathcal{U}$, such that the state transition matrix $J_{P} e^{\left(A_{P}+B_{P} F_{P}\right) \tau}$ is Schur stable.

Proof. If $J_{P}=0$, the proof is obvious. If $J_{P} \neq 0$, the statement is proven through the following reasoning. First, note that, since $\left(A_{P}, B_{P}\right)$ is reachable, there exists a linear map $F_{P, 1}: \mathcal{X}_{P} \rightarrow \mathcal{U}$, such that $A_{P}+B_{P} F_{P, 1}$ is cyclic with respect to one (nonzero) column of $B_{P}-$ e.g., the first column, henceforth denoted by $b_{P}$. Let $S$ be a similarity transformation such that the pair $\left(A_{P}^{\prime}+B_{P}^{\prime} F_{P, 1}^{\prime}, b_{P}^{\prime}\right)$, where $A_{P}^{\prime}+B_{P}^{\prime} F_{P, 1}^{\prime}=S^{-1}\left(A_{P}+B_{P} F_{P, 1}\right) S$ and $b_{P}^{\prime}=S^{-1} b_{P}$, is in controllable canonical form. Moreover, let the linear map $F_{P, 2}: \mathcal{X}_{P} \rightarrow \mathcal{U}$ be such that the spectrum of $A_{P}+B_{P} F_{P, 1}+B_{P} F_{P, 2}$ is assigned on the negative real axis as $\left\{\lambda_{i}: \lambda_{i}=-\lambda-i+1, \lambda>0, i=1,2, \ldots, n_{P}\right\}$, and, with respect to the new coordinates,

$$
\begin{aligned}
& A_{P}^{\prime}+B_{P}^{\prime} F_{P, 1}^{\prime}+B_{P}^{\prime} F_{P, 2}^{\prime}= \\
& S^{-1}\left(A_{P}+B_{P} F_{P, 1}+B_{P} F_{P, 2}\right) S,
\end{aligned}
$$

is in companion form - the two requirements are compatible since $A_{P}^{\prime}+B_{P}^{\prime} F_{P, 1}^{\prime}$ is cyclic with respect to the first column of $B_{P}^{\prime}$. Let $D=\operatorname{diag}\left\{\lambda_{1}, \ldots, \lambda_{n_{P}}\right\}$ and

$$
Q=\left[\begin{array}{cccc}
1 & 1 & \ldots & 1 \\
\lambda_{1} & \lambda_{2} & \ldots & \lambda_{n_{P}} \\
\vdots & \vdots & & \vdots \\
\lambda_{1}^{n_{P}-1} & \lambda_{2}^{n_{P}-1} & \ldots & \lambda_{n_{P}}^{n_{P}-1}
\end{array}\right]
$$

so that

$$
A_{P}^{\prime}+B_{P}^{\prime} F_{P, 1}^{\prime}+B_{P}^{\prime} F_{P, 2}^{\prime}=Q D Q^{-1} .
$$

Hence,

$$
A_{P}+B_{P} F_{P}=S Q D Q^{-1} S^{-1}
$$

follows from (A.1) and (A.2), with $F_{P}=F_{P, 1}+F_{P, 2}$. From (A.3), it follows that

$$
e^{\left(A_{P}+B_{P} F_{P}\right) \tau}=S Q e^{D \tau} Q^{-1} S^{-1},
$$

for any positive real constant $\tau$. Thus, (A.4) implies that

$$
\left\|e^{\left(A_{P}+B_{P} F_{P}\right) \tau}\right\| \leq\|S\|\|Q\|\left\|e^{D \tau}\right\|\left\|Q^{-1}\right\|\left\|S^{-1}\right\|,
$$

for any positive real constant $\tau$. Note that $\|S\|$ and $\left\|S^{-1}\right\|$ in (A.5) are positive real constant, while $\left\|e^{D \tau}\right\|,\|Q\|$, and $\left\|Q^{-1}\right\|$ only depend on $\lambda$, by virtue of the eigenvalue assignment. In particular,

$$
\left\|e^{D \tau}\right\| \leq e^{-\lambda \tau}
$$

since $-\lambda=\lambda_{1}$ is the maximum eigenvalue of the assigned set, while

$$
\|Q\| \leq\|Q\|_{\mathscr{F}}, \quad\left\|Q^{-1}\right\| \leq\left\|Q^{-1}\right\|_{\mathscr{F}}
$$

Note that the use of the Frobenius norm in the above inequalities is motivated by the need to limit the complexity of such expressions, which will be used in computations aimed at finding suitable values for $\lambda$. In light of (A.6) and (A.7), (A.5) shows that $\left\|e^{\left(A_{P}+B_{P} F_{P}\right) \tau}\right\|$ can be made arbitrarily small by picking a sufficiently large $\lambda$. In fact, in the worst case, as $\lambda$ increases, the product $\|Q\|\left\|Q^{-1}\right\|$ increases as a power of $\lambda$, while $\left\|e^{D \tau}\right\|$ decreases exponentially. On these premises, the remainder of the proof is aimed at showing that the spectrum of $J_{P} e^{\left(A_{P}+B_{P} F_{P}\right) \tau}$ can be assigned inside the open unit disc of the complex plane by a suitable choice of the linear map $F_{P}$. Hence, let $F_{P}$ be such that

$$
\left\|e^{\left(A_{P}+B_{P} F_{P}\right) \tau} x\right\|<\left\|J_{P}\right\|^{-1}\|x\|, \forall x \in \mathbb{R}^{n}, x \neq 0,
$$

which can be done since $\left(A_{P}, B_{P}\right)$ is reachable, as was shown in the first part of this proof. Then, in light of (A.8), the inequality $\left\|J_{P} e^{\left(A_{P}+B_{P} F_{P}\right) \tau} x\right\| \leq$ $\left\|J_{P}\right\|\left\|e^{\left(A_{P}+B_{P} F_{P}\right) \tau} x\right\|, \quad$ for all $x \in \mathbb{R}^{n}$, implies $\left\|J_{P} e^{\left(A_{P}+B_{P} F_{P}\right) \tau} x\right\|<\|x\|$, for all $x \in \mathbb{R}^{n}$ and $x \neq 0$, or, equivalently,

$$
\left\|J_{P} e^{\left(A_{P}+B_{P} F_{P}\right) \tau} x\right\| /\|x\|<1, \forall x \in \mathbb{R}^{n}, x \neq 0 .
$$

Hence, in particular, (A.9) implies $\sup _{x \in \mathbb{R}^{n}, x \neq 0}\left\{\left\|J_{P} e^{\left(A_{P}+B_{P} F_{P}\right) \tau} x\right\| /\|x\|\right\}<1$, or, by definition of matrix 2-norm,

$$
\left\|J_{P} e^{\left(A_{P}+B_{P} F_{P}\right) \tau}\right\|<1 .
$$

On the other hand,

$$
\left\|J_{P} e^{\left(A_{P}+B_{P} F_{P}\right) \tau}\right\|=\sigma_{M}
$$

where $\sigma_{M}$ is the maximal singular value of $J_{P} e^{\left(A_{P}+B_{P} F_{P}\right) \tau}$. Hence, (A.10) and (A.11) imply that $J_{P} e^{\left(A_{P}+B_{P} F_{P}\right) \tau}$ is Schur stable.

Proposition 7 Consider the hybrid extended system $\Sigma$. Let $(A, E)$ be observable. Then, there exists a linear map $G: \mathcal{Y} \rightarrow \mathcal{X}$, such that the state transition matrix $J e^{(A+G E) \tau}$ is Schur stable.

Proof. It can be derived from that of Proposition 6 by duality arguments.

\section{Acknowledgements}

The authors gratefully acknowledge the anonymous reviewers for their constructive comments. 


\section{References}

Basile, G., Marro, G., 1992. Controlled and Conditioned Invariants in Linear System Theory. Prentice Hall, Englewood Cliffs, New Jersey.

URL http://www3.deis.unibo.it/staff/ FullProf/GiovanniMarro/geometric.htm

Carnevale, D., Galeani, S., Menini, L., 2012a. Output regulation for a class of linear hybrid systems. Part 1: Trajectory generation. In: 51st IEEE Conference on Decision and Control. Maui, HI, USA, pp. 6151-6156.

Carnevale, D., Galeani, S., Menini, L., 2012b. Output regulation for a class of linear hybrid systems. Part 2: Stabilization. In: 51st IEEE Conference on Decision and Control. Maui, HI, USA, pp. 6157-6162.

Carnevale, D., Galeani, S., Menini, L., Sassano, M., 2014a. Output regulation of hybrid linear systems with unpredictable jumps. In: 19th World Congress of the International Federation of Automatic Control. Cape Town, South Africa, pp. 1531-1536.

Carnevale, D., Galeani, S., Menini, L., Sassano, M., 2016. Hybrid output regulation for linear systems with periodic jumps: Solvability conditions, structural implications and semi-classical solutions. IEEE Transactions on Automatic Control 61 (9), 2416-2431.

Carnevale, D., Galeani, S., Sassano, M., 2013. Necessary and sufficient conditions for output regulation in a class of hybrid linear systems. In: 52nd IEEE Conference on Decision and Control. Florence, Italy, pp. 2659-2664.

Carnevale, D., Galeani, S., Sassano, M., 2014b. A linear quadratic approach to linear time invariant stabilization for a class of hybrid systems. In: 22nd Mediterranean Conference on Control and Automation. Palermo, Italy, pp. 545-550.

Conte, G., Perdon, A. M., Zattoni, E., 2012. A geometric approach to the general autonomous regulator problem in the time-delay framework. Systems and Control Letters 61 (4), 602-608.

Francis, B., Sebakhy, O. A., Wonham, W. M., 1974. Synthesis of multivariable regulators: The internal model principle. Applied Mathematics and Optimization 1 (1), 64-86.

Francis, B. A., 1977. The linear multivariable regulator problem. SIAM Journal on Control and Optimization 15 (3), 486-505.

Goebel, R., Sanfelice, R. G., Teel, A. R., 2009. Hybrid dynamical systems. IEEE Control Systems Magazine 29 (2), 28-93.

Goebel, R., Sanfelice, R. G., Teel, A. R., 2012. Hybrid Dynamical Systems: Modeling, Stability, and Robustness. Princeton University Press, Princeton, New Jersey.

Marconi, L., Teel, A. R., 2010. A note about hybrid linear regulation. In: 49th IEEE Conference on Decision and Control. Atlanta, GA, pp. 1540-1545.

Marconi, L., Teel, A. R., 2013. Internal model principle for linear systems with periodic state jumps. IEEE Transactions on Automatic Control 58 (11), 2788-2802.

Marro, G., 1996. Multivariable regulation in geometric terms: Old and new results. In: Colloquium on Automatic Control. Vol. 215 of Lecture Notes in Control and Information Sciences. Springer-Verlag, London, pp. 77-138.

Medina, E. A., 2007. Linear impulsive systems: A geometric approach. Ph.D. thesis, Ohio State University, School of Engineering and Computer Science, Columbus, $\mathrm{OH}$, USA.

Medina, E. A., Lawrence, D. A., 2009. State feedback stabilization of linear impulsive systems. Automatica 45 (6), 1476-1480.

Perdon, A. M., Conte, G., Zattoni, E., 2015. The disturbance decoupling problem for jumping hybrid systems. In: 54th IEEE Conference on Decision and Control. Osaka, Japan, pp. 1589-1594.

Perdon, A. M., Zattoni, E., Conte, G., 2016. Disturbance decoupling with stability for linear impulsive systems. In: 6th IFAC Symposium on System Structure and Control. Vol. 49 of IFAC-PapersOnLine. Istanbul, Turkey, pp. 1-6.

Wonham, W. M., 1985. Linear Multivariable Control: A Geometric Approach, 3rd Edition. Springer-Verlag, New York.

Zattoni, E., 2008. Perfect elimination of regulation transients in discrete-time LPV systems via internally stabilizable robust controlled invariant subspaces. IEEE Transactions on Automatic Control 53 (6), 1509-1515.

Zattoni, E., Perdon, A. M., Conte, G., 2013. The output regulation problem with stability for linear switching systems: A geometric approach. Automatica 49 (10), 2953-2962. 